\title{
Trapeziidae Miers, 1886 and Tetraliidae Castro, Ng and Ahyong, 2004 (Crustacea, Brachyura): coral crabs of Western Australia, with notes on their biogeography
}

\author{
Peter Castro $^{1}$ and Melissa M. Titelius ${ }^{2}$ \\ ${ }^{1}$ Biological Sciences Department, California State Polytechnic University, \\ Pomona, CA 91768, U.S.A. \\ email: pcastro@csupomona.edu \\ 2 Department of Aquatic Zoology, Western Australian Museum, Locked Bag 49, \\ Welshpool DC, 6986, Western Australia, Australia. \\ email: melissa.titelius@museum.wa.gov.au
}

\begin{abstract}
Nineteen species in four genera of coral crabs (Trapeziidae and Tetraliidae) are listed as associated with reef corals and other colonial anthozoans in Western Australia: two Quadrella species, nine Trapezia, six Tetralia, and two Tetraloides. Although species diversity of coral crabs decreases with latitude along the coast of Western Australia, it remains relatively high in the low-diversity, high latitude coral communities along their southernmost limit on the southwestern coast.
\end{abstract}

\section{INTRODUCTION}

Brachyuran crabs belonging to the Trapeziidae and Tetraliidae are obligate symbionts of reef corals and other colonial cnidarians. Species of Trapezia are associated with reef-building, hermatypic corals of the family Pocilloporidae, and tetraliids (Tetralia and Tetraloides) with similar corals of the Acroporidae.

Collections of trapeziids and tetraliids from Western Australia, the Cocos (Keeling) Islands and Christmas Island deposited in the Western Australian Museum and other museums, as well as observations on live material, are reported herein. Specimens examined are listed starting with those from the most northerly locations. The synonymies are mostly restricted to recent nomenclatorial changes. More complete synonymies, as well as other references for many of the species listed, were given by Castro (1999a) and in the revision of Castro et al. (2004). Trapeziids and tetraliids of eastern Australia were listed by Castro (1997a) and records for all Australian regions by Davie (2002).

\section{MATERIAL AND METHODS}

The material examined is deposited in the following museums: AM (Australian Museum, Sydney), QM (Queensland Museum, Brisbane), MNHN (Muséum National d'Histoire Naturelle, Paris), WAM (Western Australian Museum, Perth), and RMNH (Nationaal Natuurhistorisch Museum, Leiden, The Netherlands).
Abbreviations used in the test are as follows: Arch. for Archipelago; coll. for collected by; I. for Island; juv. for juvenile; $\mathrm{n}$. mls for nautical miles; $\mathrm{Pt}$ for Point.

\section{SYSTEMATICS}

Family Trapeziidae Miers, 1886

Quadrella Dana, 1851

Quadrella coronata Dana, $1852 *$

Western Australia records

Galil, 1986b: 282 (off Port Hedland). - Davie, 2002: 495 (NW coast).

\section{Material examined}

Western Australia. WAM C 15818 (1 male, 1 female), $71 \mathrm{n}$. mls. NNW of Dampier Arch. $\left(19^{\circ} 30.2^{\prime} \mathrm{S}, 116^{\circ} 28.3^{\prime} \mathrm{E}\right.$ to $\left.19^{\circ} 31.3^{\prime} \mathrm{S}, 116^{\circ} 27.8^{\prime} \mathrm{E}\right)$, $86-$ $100 \mathrm{~m}$, coll. F.R.V. Soela, 01.10.1982; WAM C 15817 (1 male), $7 \mathrm{n}$. mls $\mathrm{N}$ of North West Cape, on alcyonacean, coll. R. Rowe on F.R.V. Jurabi, 05.08.1973.

\section{Remarks}

This species is associated with alcyonaceans, antipatharians and gorgonians from the Red Sea, Indian Ocean, and western Pacific Ocean (Castro 1999a; 2000). In Australia, the species has only been recorded from Western Australia (Davie 2002). 


\section{Quadrella maculosa Alcock, 1898}

\section{Western Australia records}

Davie, 2002: 495 (NW coast).

\section{Material examined}

Western Australia. WAM C 16790 (1 male), 106 n. mls $N$ of Port Hedland (18 $8^{\circ} 30.1^{\prime} S, 118^{\circ} 36.2^{\prime} \mathrm{E}$ to $\left.18^{\circ} 31.6^{\prime} \mathrm{S}, 118^{\circ} 37^{\prime} \mathrm{E}\right), 136-146 \mathrm{~m}$, coll. F.R.V. Soela, 19.08.1982; WAM C 24497 (2 females), 18³8'S, $118^{\circ} 38^{\prime} \mathrm{E}, 132-135 \mathrm{~m}$, coll. F.R.V. Courageous, 28.05.1978; WAM C 15988 (1 female), unknown station, coll. F.R.V. Courageous, 1978; WAM C 15980 (2 females), $80 \mathrm{n}$. mls NW of Port Hedland $\left(19^{\circ} 10^{\prime} \mathrm{S}, 117^{\circ} 49^{\prime} \mathrm{E}\right.$ to $\left.19^{\circ} 07^{\prime} \mathrm{S}, 117^{\circ} 50^{\prime} \mathrm{E}\right)$, coll. F.R.V. Soela, 18.08.1982.

\section{Remarks}

Known inhabiting antipatharians from the Red Sea and western Indian Ocean and in the Pacific Ocean from Japan to French Polynesia (Castro 1999a; 2000) and Western Australia (Davie 2002).

\section{Trapezia Latreille, 1828}

\section{Trapezia cymodoce (Herbst, 1801)}

\section{Western Australian records}

Balss, 1935: 139, 145 (Shark Bay), 146 (Rottnest I.); Black and Prince, 1983: 140 (Pt Quobba; Rottnest I.); Berry and Morgan, 1986: 61 (Scott Reef, as Trapezia cf. cymodoce); Jones, 1990: 201 (Shark Bay); Morgan, 1990: 51 (Kimberley region); Jones and Morgan, 1993: 156 (Rottnest I.); Davie, 2002: 498, 499 (NW, central, and lower W coast).

\section{Material examined}

Western Australia. QM W 17771 (1 juv. female), Hibernia Reef $\left(11^{\circ} 58.3^{\prime} \mathrm{S}, 123^{\circ} 32.8^{\prime} \mathrm{E}\right)$, patch reef, 6.0$9.0 \mathrm{~m}$, coll. J. Short, 11.05.1992; QM W 17801 (1 male, 1 female), $6.0 \mathrm{~m}$, on Stylophora pistillata, 12.05.1992; $11^{\circ} 58.7^{\prime} \mathrm{S}, 123^{\circ} 22.8^{\prime} \mathrm{E}, 1$ male, 1 female, $6.0 \mathrm{~m}$, 12.05.1992 (QM W 17809); QM W 17638 (1 male, 1 female), Cartier Reef, reef slope (12³0.7'S, $\left.123^{\circ} 33.4^{\prime} \mathrm{E}\right), 23.0 \mathrm{~m}$, on Seriatopora sp., J. Short, 07.05.1992; QM W 15760; (2 males, 2 females), Cartier Reef, lagoon $\left(12^{\circ} 31.8^{\prime} \mathrm{S}, 123^{\circ} 33.1^{\prime} \mathrm{E}\right), 4.0-6.0 \mathrm{~m}, \mathrm{~J}$. Short, 05.05.1992; QM W 21106 (1 female), stn 5, Jones I., Vansittart Bay, Kimberley region $\left(13^{\circ} 44.5^{\prime} \mathrm{S}\right.$, $\left.126^{\circ} 22.2^{\prime} \mathrm{E}\right)$, coll. J. Short, 22.11.1995; WAM C 20581 (1 feminised male, 1 female), Cassini I. (13 ${ }^{\circ} 56^{\prime} \mathrm{S}$, 1253'ㄹ), 4.0-24.0 m, coll. KIRE 1991, 17-18.08.1991; WAM C 24478 (1 female), Scott Reef (14\%03'S, $\left.121^{\circ} 46^{\prime} \mathrm{E}\right)$, coll. K. Kenneally, 27.08.1977; WAM C 18891 ( 1 male, 1 female), stn 54, Condillac I. (14º7'S, $\left.125^{\circ} 34^{\prime} \mathrm{E}\right), 10.0 \mathrm{~m}, \mathrm{G}$. Morgan, 16.07.1988; WAM C 20592 (1 male), KIRE 1991 stn 19, SE end of Condillac I. (1406'S, $\left.125^{\circ} 33^{\prime} \mathrm{E}\right)$, coll. G. Morgan, 18.08.1991; WAM C 20557 (1 male, 1 female), KIRE 1991 stn 20,
SW of Fenelon I. $\left(14^{\circ} 09^{\prime} \mathrm{S}, 125^{\circ} 39^{\prime} \mathrm{E}\right), 4.0-14.0 \mathrm{~m}$, coll. G. Morgan, 19.08.1991; WAM C 20577 (1 male, 1 female), KIRE 1991 stn 24, Albert Reef (14¹5'S, $\left.125^{\circ} 10^{\prime} \mathrm{E}\right), 3.0-15.0 \mathrm{~m}$, coll. G. Morgan, 20.08.1991; WAM C 21814 (1 male, 1 female), Monte Bello Is, channel between Alpha and Trimoville Is $\left(20^{\circ} 24^{\prime} \mathrm{S}\right.$, $\left.115^{\circ} 33^{\prime} \mathrm{E}\right), 6.0 \mathrm{~m}$, coll. S. Slack-Smith and P. Berry, 23.08.1993; WAM C 27501 (7 males, 7 females), DA4/ 00/14, NW of Legendre I., Dampier Archipelago (2021.206'S, $\left.116^{\circ} 50.439^{\prime} \mathrm{E}\right), \quad 9.0-10.0 \mathrm{~m}$, on Pocillopora verrucosa, coll. P. Castro, 30.07.2000; WAM C 27508 (2 males, 2 females), DA4/00/37, Legendre I. (20²1.270'S, $\left.116^{\circ} 50.557^{\prime} \mathrm{E}\right), 7.5 \mathrm{~m}$, on $P$. damicomis, coll. P. Castro and C. Bryce, 06.08.2000; WAM C 27515 (1 male), Legendre I. (20²3.354'S, $\left.116^{\circ} 53.802^{\prime} \mathrm{E}\right), \mathrm{DA} 4 / 00 / 38$, on $P$. verrucosa, coll. $\mathrm{P}$. Castro and G. Morgan, 06.08.2000; WAM C 27491 (3 males, 5 females), DA4/00/16, NW of Legendre I. (20²4.025'S, $\left.116^{\circ} 55.079 ' \mathrm{E}\right), 13-14 \mathrm{~m}$, on $P$. verrucosa, coll. P. Castro, 31.07.2000; WAM C 24646 (1 male, 1 female), stn DA1/98/03, Legendre I. (20²4.320'S, $\left.116^{\circ} 108^{\prime} \mathrm{E}\right), 2.0-15.0 \mathrm{~m}$, coll. M. Titelius et al., 18.10.1998; WAM C 28115 (1 male), stn DA1/18/98, Haüy I. (20²6.400'S, $\left.116^{\circ} 58.634^{\prime} \mathrm{E}\right), 3.0-10.0 \mathrm{~m}$, coll. M. Titelius et al., 23.10.1998; WAM C 27507 (1 male, 1 female), DA4/00/34, W of Gidley I. (20²7.695'S, $\left.116^{\circ} 47.512^{\prime} \mathrm{E}\right), 10.0 \mathrm{~m}, \mathrm{P}$. damicornis, coll. P. Castro and C. Bryce, 05.08.2000; WAM C 25573 (1 male, 1 female), stn DA1/98/29, Angel I. (20²9.18'S, $\left.116^{\circ} 47.71^{\prime} \mathrm{E}\right)$, coll. M. Titelius et al., 27.10.1998;WAM C 15979 (1 male, 1 female), Kendrew I., reef flat $\left(20^{\circ} 29^{\prime} \mathrm{S}, 116^{\circ} 32^{\prime} \mathrm{E}\right)$, coll. L. Marsh and R. George, 11.10.1972; WAM C 16007 (1 female), Crown-ofThorns Survey, Kendrew I., 20.02.1973; WAM C 16008 (1 male), Crown-of-Thorns Survey, Kendrew I., 22.02.1973; WAM C 16006 (1 male, 1 female), Crown-of-Thorns Survey, Kendrew I., 28.02.1973; WAM C 16023 (1 male, 2 females), Crown-of-Thorns Survey, Kendrew I., 11.05.1973; WAM C 15931 (4 males, 5 females), WAM C 11879 ( 1 male, 1 female), Crown-of-Thorns Survey, 20.05.1974; 7.5 m; WAM C 28116 (2 juvs), stn DA3/99/46, Kendrew I. (20²9.671'S, $\left.116^{\circ} 35.894^{\prime} \mathrm{E}\right), 3.0-11.0 \mathrm{~m}$, coll. M. Titelius et al., 30.08.1999; WAM C 28117 (1 male), stn DA3/99/44, Rosemary I. (20²9.629'S, $\left.116^{\circ} 34.425^{\prime} \mathrm{E}\right)$, $2.5-6.0 \mathrm{~m}$, on $P$. damicornis, coll. M. Titelius et al. 28.08.1999; WAM C 27495 (4 males, 4 females), DA4/ 00/23, NW corner of Roly Rock (20²9.88'S, $\left.116^{\circ} 30.05^{\prime} \mathrm{E}\right), 15.0 \mathrm{~m}$, on $P$. verrucosa, coll. P. Castro, 02.08.2000; WAM C 24483 (2 males, 2 females), Mariel King Expedition, NE Malus Is. (20³1'S, $116^{\circ} 41$ 'E), coll. R.D. Royce, 31.05.1960; WAM C 16020 ( 1 male, 1 female), WSW of Kendrew I. (2032'S, 116 $\left.{ }^{\circ} 38^{\prime} \mathrm{E}\right)$, coll. L. Marsh, 26.05.1972; WAM C 28118 ( 1 male, 1 female) stn DA3/99/49, Goodwyn I. $\left(20^{\circ} 32.398^{\prime} \mathrm{S}, 116^{\circ} 33.443^{\prime} \mathrm{E}\right), 3.0-9.0 \mathrm{~m}$, coll. M. Titelius et al., 31.08.1999; WAM C 27494 (2 males, 2 females), DA4/00/08, SW tip of West Lewis I. (20³6.310'S, $\left.116^{\circ} 35.696^{\prime} \mathrm{E}\right), 5.0 \mathrm{~m}$, on Pocillopora sp., 
coll. P. Castro 27.07.2000; WAM C 16031 (2 males, 3 females), Kendrew I., W of West Pt $\left(20^{\circ} 40^{\prime} \mathrm{S}\right.$, $\left.116^{\circ} 42^{\prime} \mathrm{E}\right)$, coll. Crown-of-Thorns Survey, 25.03.1974; WAM C 22448 (1 male), stn 6, South Muiron I. (21 $\left.{ }^{\circ} 39.45^{\prime} \mathrm{S}, 114^{\circ} 20.72^{\prime} \mathrm{E}\right), 11.0-13.0 \mathrm{~m}$, coll. M. Titelius et al., 21.08.1995; WAM C 22449(1 female), stn 7, South Muiron I., 1.0-1.5 m, coll. M. Titelius et al., 22.08.1995; WAM C 22450 (1 female), stn 8, South Muiron I., $4.0-8.0 \mathrm{~m}$, coll. M. Titelius et al., 22.08.1995; WAM C 22453 (1 male), stn 8, South Muiron I., $11.0-13.0 \mathrm{~m}$, on $P$. eydouxi, coll. $\mathrm{M}$. Titelius et al.; WAM C 22452 (1 male, 1 female), stn 6 , SE of South Muiron I. $\left(21^{\circ} 40.86^{\prime} \mathrm{S}, 114^{\circ} 20.55^{\prime} \mathrm{E}\right)$, 4.0-8.0 m, coll. M. Titelius et al. 21.08.1995; WAM C 23045 (1 female), stn 4 , SW of South Muiron I.

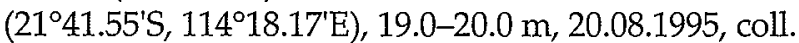
M. Titelius et al.; WAM C 16828 ( 1 male, 1 female), North West Cape, S of Sandy Bay (21⒋'S, $\left.114^{\circ} 10^{\prime} \mathrm{E}\right)$, 6.0-9.0 m, 31.05.1981; WAM C 24470 (1 male), stn 2, on Seriatopora sp., coll. P. Berry, 27.05.1981; WAM C 15941 (2 males, 2 females), Ningaloo, $14 \mathrm{mi}$. $\mathrm{N}$ of $\mathrm{Pt}$ Cloates $\left(22^{\circ} 42^{\prime} \mathrm{S}, 113^{\circ} 40^{\prime} \mathrm{E}\right), 6.0 \mathrm{~m}$, coll. Ningaloo Expedition 08.09.1968; ex AM P 45107 (1 male), W of Pt Cloates (22 $\left.42^{\prime} \mathrm{S}, 113^{\circ} 39^{\prime} \mathrm{E}\right), 1.0 \mathrm{~m}$, on Acropora sp., 30.08.1968; WAM C 15989 (1 female), stn 19, Warroora $\left(23^{\circ} 29^{\prime} \mathrm{S}, 113^{\circ} 48^{\prime} \mathrm{E}\right)$, coll. L. Marsh, 5.06.1981; WAM C 15944 (1 female), Pt Quobba $\left(24^{\circ} 29^{\prime} \mathrm{S}, 113^{\circ} 25^{\prime} \mathrm{E}\right)$, coll. G. Mees, 16.12.1962; WAM C 22447 ( 1 female), stn 24, Shark Bay, $N$ end of Bernier I. $\left(24^{\circ} 43.34^{\prime} \mathrm{S}, 113^{\circ} 09.45^{\prime} \mathrm{E}\right), 1.0-16.0 \mathrm{~m}, 19.05 .1995$; WAM C 22445 ( 1 male), stn 17 (24\%59.24'S, $\left.113^{\circ} 07.18^{\prime} \mathrm{E}\right), 1.0-5.0 \mathrm{~m}, 17.05 .1995$; WAM C 15945 (1 male, 1 female), Carnarvon $\left(24^{\circ} 53^{\prime} \mathrm{S}, 113^{\circ} 40^{\prime} \mathrm{E}\right), 10$ 12.1959; WAM C 15937 (1 female), Carnarvon

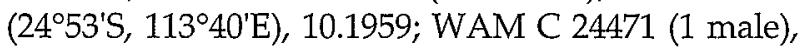
stn 1, Shark Bay, NE of Dorre I. (245․ $7^{\prime}$ ', $\left.113^{\circ} 07.3^{\prime} \mathrm{E}\right)$, coll. M. Titelius et al. 11.05.1995; WAM C 22446 (1 female), stn 13, Shark Bay, W of Dorre I (2509.04'S, $\left.113^{\circ} 05.33^{\prime} \mathrm{E}\right), 5.0-22.0 \mathrm{~m}, 16.05 .1995$; WAM C 7365-70 (1 male, 5 females), Dirk Hartog I. (25⒉'S, 112 $\left.{ }^{\circ} 57^{\prime} \mathrm{E}\right)$, coll. B. Wilson; WAM C 15929 (1

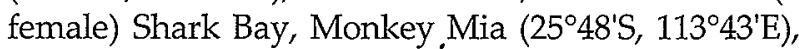
coll. A. Kalmins, 25-30.08.1960; WAM C 20150 (1 male, 1 female), Shark Bay, South Passage $\left(26^{\circ} 08^{\prime} \mathrm{S}\right.$, $\left.113^{\circ} 10^{\prime} \mathrm{E}\right)$, coll. G. Morgan, 13.03.1986; WAM C 20191 (2 males, 2 females), WAM C 20178 (4 males, 2 females), stn 9, Shark Bay, Monkey Rock (26이, $\left.113^{\circ} 10^{\prime} \mathrm{E}\right)$, coll. G. Morgan, 11.03.1986; WAM C 15940 ( 1 male, 2 females), Port Gregory, reef flat, $\left(28^{\circ} 12^{\prime} \mathrm{S}\right.$, $\left.114^{\circ} 15^{\prime} \mathrm{E}\right), 27.12 .1962$, coll. B.R. Wilson and R. SlackSmith; WAM C 15946, WAM C 15928 (2 males, 3 females), Port Gregory $1.0 \mathrm{~m}, 26.12 .1962$; WAM C 24476 ( 1 male) Houtman Abrolhos Is, coll. J. Allchin, 03-08.1960; WAM C 11735 (2 females), Beacon I. $\left(28^{\circ} 28^{\prime} \mathrm{S}, 113^{\circ} 47^{\prime} \mathrm{E}\right)$, coll. L. Marsh et al., 22.04.1974; WAM C 11742 (1 male, 1 female), Beacon I., coll. S. Bingham, 21.04.1974; WAM C 24481 (1 male, 1 female), NW of Split I., Morning Reef $\left(28^{\circ} 28.1^{\prime} \mathrm{S}\right.$, $113^{\circ} 47.8^{\prime} \mathrm{E}$ ), on $P$. damicornis, coll. C. Bryce,
08.04.1978; WAM C 11931 (1 male, 1 female), stn D 23, Leo I. (28 $\left.41^{\prime} \mathrm{S}, 113^{\circ} 51^{\prime} \mathrm{E}\right)$, coll. Sixth Abrolhos Expedition, 31.08.1972; WAM C 15932 (4 males, 3 females), WAM C 15936 (2 males), Rat I., (28 $43^{\prime}$ 'S, $\left.113^{\circ} 46^{\prime} \mathrm{E}\right), 3.7 \mathrm{~m}$, coll. F.R.V. Lancelin, 15.03.1963, WAM C 11932 (1 female), stn D25, Rat I., coll. Sixth Abrolhos Expedition, 29.08.1972; WAM C 16005 (1 male), Gregory Reef, Geraldton $\left(28^{\circ} 46^{\prime} \mathrm{S}, 114^{\circ} 37^{\prime} \mathrm{E}\right)$, coll. G. Kendrick, 25.09.1962; WAM C 16028 (1 male, 1 female), $\mathrm{N}$ of Gun I. (28 $\left.53^{\prime} \mathrm{S}, 113^{\circ} 51^{\prime} \mathrm{E}\right)$, coll. A. James and G. Davenport, 05.01.1968; WAM C 15934 (1 male, 1 female), $31^{\circ} 01^{\prime} S 115^{\circ} 20^{\prime} \mathrm{E}$, coll. F.R.V. Lancelin, Neptune Submariners, 14.01.1958; WAM C 24473 ( 1 male, 3 females), Wreck Point (3130'S, $\left.115^{\circ} 35^{\prime} \mathrm{E}\right)$, coll. A. Robinson, 20.04.1958; WAM C 20373 (1 male), Rottnest I., Kitson Pt. (32 $01^{\prime}$ 'S, 115³0'E), coll. G. Morgan, 23.01.1991; WAM C 24502 (2 males), Rottnest I., Salmon Bay, (32 $01.2^{\prime} S$, $\left.115^{\circ} 31.1^{\prime} \mathrm{E}\right), 2.0 \mathrm{~m}$, on Acropora sp., coll. P. Castro and M. Titelius, 03.02.2000; WAM C 24503 (1 female), Rottnest I., Salmon Bay, (32 $01.2^{\prime} S$, $\left.115^{\circ} 31.1^{\prime} \mathrm{E}\right), 2.0 \mathrm{~m}$, on $P$. damicornis, coll. P. Castro and M. Titelius, 03.02.2000.

\section{Remarks}

A juvenile, incomplete specimen from Scott Reef originally identified as Trapezia cf. cymodoce by Berry and Morgan (1986: 61; WAM C 14120) could not be identified with certainty.

\section{Trapezia digitalis Latreille, 1828}

\section{Western Australian records}

Black and Prince, 1983: 140 (Rottnest I.); Jones and Morgan, 1993: 156 (Rottnest I.); Castro, 1999a: 107 (Cocos Keeling Is., Christmas I).

\section{Material examined}

Western Australia. WAM C 24480 (1 male, 1 female), Dampier Archipelago, WSW of Kendrew I. (20³2'S, $\left.116^{\circ} 38^{\prime} \mathrm{E}\right)$, coll. L. Marsh, 26.05.1972; WAM C 22460 (1 male, 1 female), stn 8, South Muiron I. $\left(21^{\circ} 39.45^{\prime} \mathrm{S}, 114^{\circ} 20.72^{\prime} \mathrm{E}\right), 11.0-13.0 \mathrm{~m}$, on Pocillopora eydouxi, coll. M. Titelius et al. 22.08.1995; WAM C 15974 (1 male), Houtman Abrolhos Is, Pidgin I. $\left(28^{\circ} 30^{\prime} \mathrm{S}, 113^{\circ} 45^{\prime} \mathrm{E}\right)$, coll. P. Barret Lennard, - .05.1959. Christmas Island, Indian Ocean. WAM C 15973 (1 male), S end of Flying Fish Cove, E. Car, 15.06.1961.

\section{Trapezia formosa Smith, 1869}

Western Australian records

Davie, 2002: 500 (NW coast).

\author{
Material examined \\ Western Australia. WAM C 24479 (1 female),
}


Dampier Archipelago, WSW of Kendrew I. (20³2'S, $\left.116^{\circ} 38^{\prime} \mathrm{E}\right)$, coll. L. Marsh, 26.05.1972. Christmas Island, Indian Ocean. WAM C 15943 (1 male, 3 females), Flying Fish Cove, coll. R. Slack-Smith and A. Paterson, - .09.1969; (1 male), WAM C 24084, stn 1, Flying Fish Cove, 1.0-9.0 m, coll. S. SlackSmith and L. Marsh, 15.02.1987; WAM C 24086 (1 female), Ethel Beach, 20.02.1987, coll. G. Morgan.

\section{Remarks}

The species is known throughout the Indo-west Pacific and eastern Pacific regions (Castro 1998). In Australian waters it has only been recorded from Western Australia (Davie 2002).

\section{Trapezia guttata Rüppell, 1830}

\section{Western Australian records}

Berry and Morgan, 1986: 61 (Scott Reef); Morgan, 1990: 52 (Kimberley region); Castro, 1999a: 110 (Cocos (Keeling) Is., Christmas I.); Davie, 2002: 500 (NW coast).

\section{Material examined}

Western Australia. QM W 17772 (1 male, 1 female), Hibernia Reef, patch reef (11 ${ }^{\circ} 58.3^{\prime} \mathrm{S}$, 12332.8'E), 6.0-9.0 m, coll. J. Short, 11.05.1992; QM W 17782 (1 male, 1 female), Hibernia Reef, on Seriatopora sp; QM W 17803 (1 male, 1 female), Hibernia Reef, on Stylophora sp.; QM W17667 (1 male), Cartier Reef (12 $\left.30.7^{\prime} \mathrm{S}, 123^{\circ} 33.4^{\prime} \mathrm{E}\right), 23.0 \mathrm{~m}$, on base of Pachyseris, coll. J. Short, 07.05.1992; QM W 1763 (3 females), Cartier Reef, on Seriatopora sp., coll. J. Short, 07.05.1992; QM W 17571 (1 male, 1 juv. male, 1 female, 1 juv. female), Cartier Reef, lagoon, $\left(12^{\circ} 31.8^{\prime} \mathrm{S}, 123^{\circ} 33.1^{\prime} \mathrm{E}\right), 4.0-6.0 \mathrm{~m}$, coll. J. Short, 05.05.1992; WAM C 22988 (1 male), Seringapatam Reef $\left(13^{\circ} 32.47^{\prime} \mathrm{S}, 121^{\circ} 59.9^{\prime} \mathrm{E}\right)$, coll. C. Bryce, 15.09.1996; QM W 21301 (4 males, 6 females), stn 18 , Kimberley region $\left(13^{\circ} 45.3^{\prime} \mathrm{S}, 126^{\circ} 48.5^{\prime} \mathrm{E}\right), 1.5$ $\mathrm{m}$, coll. J. Short, 29.11.1995; WAM C 20583, stn 16, W of Cassini I. (13 $\left.{ }^{\circ} 56^{\prime} S, 1^{\circ} 5^{\circ} 37^{\prime} \mathrm{E}\right)$, coll. KIRE 1991, 17-18.08.1991; WAM C 22670 (1 male, 1 female), E of Cassini I. $\left(13^{\circ} 56.36^{\prime} \mathrm{S}, 125^{\circ} 38.47^{\prime} \mathrm{E}\right)$, stn 13 , on Seriatopora sp., coll. M. Titelius et al., 28.11.1996; WAM C 18915 (1 male, 1 female), stn 58, Long Reef $\left(13^{\circ} 59^{\prime} \mathrm{S}, 125^{\circ} 44^{\prime} \mathrm{E}\right)$, to $15.0 \mathrm{~m}$, coll. G. Morgan, 17.07.1988; WAM C 14119 (6 males, 2 feminized males, 6 females), stn 1, Scott Reef, Sandy Islet $\left(14^{\circ} 03^{\prime} \mathrm{S}, 1^{\circ} 41^{\circ} \mathrm{E}\right), 2.0-18.0 \mathrm{~m}$, coll. P. Berry, 07.09.1984; WAM C 22671 (1 male, 1 female), stn 10, Albert Reef $\left(14^{\circ} 15.5^{\prime} \mathrm{S}, 125^{\circ} 10^{\prime} \mathrm{E}\right)$, on Seriatopora sp., M. Titelius et al., 27.11.1996; WAM C 20694 (1 male), stn 25, NW end of West Montalivet I. (14 $\left.{ }^{\circ} 17^{\prime} \mathrm{S}, 125^{\circ} 13^{\prime} \mathrm{E}\right), 3.0-26.0 \mathrm{~m}$, coll. KIRE 1991, 21.08.1991; WAM C 22669 (1 male, 1 female), stn 8, Rob Roy Reef $\left(14^{\circ} 25.5^{\prime} \mathrm{S}, 124^{\circ} 51.9^{\prime} \mathrm{E}\right)$, on Seriatopora sp., M. Titelius et al., 26.11.1996; RMNH D37773 (1 male), stn 49, Rob Roy Reef, coll. G. S. Morgan, 15.07.1988; WAM C 22668 (1 female), stn 19, De Freycinet I. (14'59.3'S, $\left.124^{\circ} 32.3^{\prime} \mathrm{E}\right)$, M. Titelius et al., 01.12.1996; WAM C 20513 (1 male), Beagle Reef (15'21'S, $\left.123^{\circ} 32^{\prime} \mathrm{E}\right)$, stn 35, 4.0-12.0 m, coll. KIRE 1991 expedition, 24.08.1991. Christmas. Island, Indian Ocean. (1 male), Dolly Beach, coll. S.R. Slack-Smith, -.02.1987 (WAM C 24085). Cocos (Keeling) Islands, Indian Ocean. WAM C 19750 (2 males, 2 females), $S$ end of lagoon, Blue Hole, stn 36, G. Morgan; WAM C 19697 (1 male), N of West I., $\operatorname{stn} 25$, to $30 \mathrm{~m}, 21.02 .1989$, G. Morgan.

\section{Trapezia lutea Castro, 1997}

\section{Western Australian records}

Jones and Morgan, 1994: 178-179 (colour, as Trapezia cymodoce); 2002: 178-179 (colour); Castro, 1997a: 84 (Dampier Arch., Cocos (Keeling) Is., Christmas I.), 1999a: 111 (Dampier Arch., Cocos (Keeling) Is., Christmas I.); Davie, 2002: 500 (NW coast).

\section{Material examined \\ Western Australia. QM W 17802 (1 male, 1} female), Hibernia Reef (11 $\left.58.7^{\prime} \mathrm{S}, 123^{\circ} 22.8^{\prime} \mathrm{E}\right), 6.0 \mathrm{~m}$, on Stylophora pistillata, coll. J. Short, 12.05.1992; QM W 17810 (1 male), Hibernia Reef, on Acropora sp.; QM W 17597 (6 females), Cartier Reef, reef slope, (12 $\left.{ }^{\circ} 30.7^{\prime} \mathrm{S}, 123^{\circ} 33.4^{\prime} \mathrm{S}\right), 13.0 \mathrm{~m}$, coll. J. Short 06.05.1992; QM W 17640 (2 males, 1 female), Cartier Reef, $23.0 \mathrm{~m}$, on Seriatopora sp., 07.05.1992; QM W 17682 (1 juv. male), Cartier Reef, reef slope $\left(12^{\circ} 31.4^{\prime} \mathrm{S}, 123^{\circ} 32.7^{\prime} \mathrm{E}\right), 12.0-14.0 \mathrm{~m}$, on Tubipora musica, 08.05.1992, coll. J. Short; WAM C 27510 (1 female), stn DA4/00/14, Dampier Arch., NW of Legendre I. (20²1.206'S, $\left.116^{\circ} 50.439^{\prime} \mathrm{E}\right), 9.0-10.0 \mathrm{~m}$, on Pocillopora verrucosa, coll. P. Castro, 30.07.2000; WAM C 27490 (1 female), NW of Delambre $\mathrm{I}$. $\left(20^{\circ} 25.936^{\prime} \mathrm{S}, 116^{\circ} 04.017^{\prime} \mathrm{E}\right)$, stn DA4/00/40, $5.0 \mathrm{~m}$, on Pocillopora sp., coll. P. Castro and M. Berggren, 07.08.2000; WAM C 28203 (1 male, 1 female), Crown-of-Thorns Survey, Kendrew I., (20²8.5'S, $116^{\circ} 32^{\prime} \mathrm{E}$ ), $6.0 \mathrm{~m}, 20.05 .1974$; WAM C 27498 (1 juv. male), stn DA4/00/23, NW corner of Roly Rock (20²9.88'S, $\left.116^{\circ} 30.05^{\prime} \mathrm{E}\right), 15.0 \mathrm{~m}$, on $P$. verrucosa, coll. P. Castro, 02.08.2000; WAM C 28120 ( 1 female), Malus Is. $\left(20^{\circ} 30.050^{\prime} \mathrm{S}, 116^{\circ} 40.594^{\prime} \mathrm{E}\right)$, stn DA3/99/36, $6.0-14.0 \mathrm{~m}$, on $P$. damicornis, coll. M. Titelius et al., 27.08.1999; WAM C 22451 (1 female), stn 8, South Muiron I. (2139.45'S, $\left.114^{\circ} 20.72^{\prime} \mathrm{E}\right), 11.0-13.0 \mathrm{~m}$, on $P$. eydouxi, coll. M. Titelius et al., 22.08.1995. Christmas Island, Indian Ocean. WAM C 24469 (1 female), Flying Fish Cove, coll. R. Slack-Smith and A. Paterson, -.09.1969; C 24083 (1 female), Flying Fish Cove, 10.0-15.0 m, coll. G. Morgan, 11.02.1987; WAM C 24080 (1 female), Ethel Beach, coll. G. Morgan, 20.02.1987. Cocos (Keeling) Islands, 
Indian Ocean. WAM C 19667 (1 female), stn 32, NW end of North Keeling I., to $28 \mathrm{~m}$, coll. G. Morgan, 23.02.1989; WAM C 19820 (1 male, 1 female), stn 13, Landing Place, North Keeling I., to $29 \mathrm{~m}$, coll. G. Morgan, 17.02.1989; WAM C 19650 (1 male, 1 female), stn 4, Horsburgh I., to $37 \mathrm{~m}$, coll. G. Morgan, 09.02.1989; WAM C 19666 (1 male, 3 females), $\sin 7, \mathrm{NW}$ end of Direction I., to $18 \mathrm{~m}$, coll. G. Morgan, 10.02.1989; WAM C 19955 (1 male, 1 female), Home I., stn 27, coll. L. Marsh, 21.02.1989; WAM C 19487 (1 female), West I., stn 1, coll. G. Morgan, 08.02.1989; WAM C 19698 (1 male, 1 female), $\operatorname{stn} 25, \mathrm{~N}$ of West I., to $30 \mathrm{~m}$, coll. $\mathrm{G}$. Morgan, 21.02.1989; WAM C 19813 (1 male, 1 female), stn 24, SW end of West I., reef flat, coll. L. Marsh, 20.02.1989.

\section{Remarks}

The colour pattern of live material was similar to that of western Pacific Ocean populations (see Castro 1997a: 86, Castro 1999b: 53). It differed from eastern Indian Ocean populations by the lack of a purple-brown spot at the distal end of the propodi of the walking legs (Castro 1999a: 112).

\section{Trapezia plana Ward, 1941}

Trapezia punctipes Castro, 1997a: 87, figs 2C, 2D, 3, pls $2 \mathrm{C}, 5 \mathrm{~A}$.

Trapezia plana - Castro, 2002: 364.

\section{Western Australian records}

Davie, 2002: 500 (NW coast, as Trapezia punctipes).

\section{Material examined}

Western Australia. QM W 17808 (1 female), Hibernia Reef $\left(11^{\circ} 58.7^{\prime} \mathrm{S}, 123^{\circ} 22.8^{\prime} \mathrm{E}\right)$, patch reef, 6.0 $\mathrm{m}$, coll. J. Short, 12.05.1992; QM W 17644 (1 male, 1 female), Cartier Reef $\left(12^{\circ} 30.7^{\prime} \mathrm{S}, 123^{\circ} 33.4^{\prime} \mathrm{E}\right)$, reef slope, $23 \mathrm{~m}$, on Seriatopora sp., coll. J. Short, 07.05.1992.

\section{Remarks}

Trapezia plana is known from the Andaman Sea and the western Pacific from the Mariana Is to Fiji. Its status was reviewed by Castro (2002).

\section{Trapezia septata Dana, 1852}

\section{Western Australian records}

Black and Prince, 1983: 140 (Rottnest I., as Trapezia areolata); Berry and Morgan, 1986: 61 (Scott Reef); Morgan, 1991: 52; Jones and Morgan, 1993: 156 (Rottnest I.); Castro, 1999a: 114 (Cocos (Keeling) Is.); Davie, 2002: 501 (NW, central, and lower west coast).

\section{Material examined}

Western Australia. QM W 17944 (1 male), Hibernia Reef $\left(11^{\circ} 59^{\prime} \mathrm{S}, 123^{\circ} 22^{\prime} \mathrm{E}\right), 0.0-0.5 \mathrm{~m}$, coll. J. Short, 15.05.1992; QM W 17673 (1 male), Cartier Reef, reef slope $\left(12^{\circ} 31.4^{\prime} \mathrm{S}, 123^{\circ} 32.7^{\prime} \mathrm{E}\right), 12.0-14.0 \mathrm{~m}$, on Tubipora musica, coll. J. Short, 08.05.1992; QM W 17601 (1 male, 2 females), Cartier Reef $\left(12^{\circ} 32.3^{\prime} \mathrm{S}, 123^{\circ} 33.4^{\prime} \mathrm{E}\right), 13.0 \mathrm{~m}, 06.05 .1992$; WAM C 20582 (1 male), Kimberley region, Cassini $\mathrm{I}$. (135' $\left.\mathrm{S}, 125^{\circ} 37^{\prime} \mathrm{E}\right), 4.0-24.0 \mathrm{~m}$, coll. KIRE 1991, 1718.08.1991; WAM C 18901 (1 male), stn 58, Long Reef (13⒌ $\left.9^{\prime} \mathrm{S}, 125^{\circ} 44^{\prime} \mathrm{E}\right)$, to $15 \mathrm{~m}$, coll. G. Morgan, 17.07.1988; WAM C 14118 (1 male, 1 female), stn 1 , Scott Reef, Sandy Islet (14 $\left.{ }^{\circ} 03^{\prime} \mathrm{S}, 121^{\circ} 46^{\prime} \mathrm{E}\right), 2.0-18.0$ m, coll. P. Berry, 07.09.1984; WAM C 27514 (3 males, 6 females), DA4/00/14, Dampier Arch., NW of Legendre I. $\left(20^{\circ} 21.206^{\prime} \mathrm{S}, 116^{\circ} 50.439^{\prime} \mathrm{E}\right), 9.0-10.0$ $\mathrm{m}$, on Pocillopora verrucosa, coll. P. Castro, 30.07.2000; WAM C 27492 (1 male), stn DA4/00/38, Legendre I. $\left(20^{\circ} 23.354^{\prime} S, 116^{\circ} 53.802^{\prime} \mathrm{E}\right)$, on $P$. verrucosa, coll. P. Castro and G. Morgan, 06.08.2000; WAM C 28121 (1 female), stn DA1/98/ 03, Legendre I. (20²4.320'S, $\left.116^{\circ} 56^{\prime} \mathrm{E}\right), 2.0-15.0 \mathrm{~m}$, coll. M. Titelius et al. 18.10.1998; WAM C 11873 (1 female), Kendrew I. (20³8.3'S, 116 $\left.32^{\circ} \mathrm{E}\right)$, coll. Crown-of-Thorns Survey, 11.05.1973; WAM C 22458 (1 female), stn 8, South Muiron I. (21 $39.45^{\prime} S$, $\left.114^{\circ} 20.72^{\prime} \mathrm{E}\right), 11-13 \mathrm{~m}$, on $P$. eydouxi, coll. $\mathrm{M}$. Titelius et al., 22.08.1995; WAM C 15976 (1 female), Pt Quobba $\left(24^{\circ} 29^{\prime} S, 113^{\circ} 25^{\prime} \mathrm{E}\right)$, coll. G. Mees, 16.12.1962; WAM C 22457 (1 female), stn 14, Shark Bay, Dorre I. $\left(25^{\circ} 16.38^{\prime} \mathrm{E}, 113^{\circ} 05.65^{\prime} \mathrm{E}\right), 3.0-20.0 \mathrm{~m}$, 16.05.1995; WAM C 11737 ( 1 male, 1 female), Houtman Abrolhos Is., $S$ of Beacon I. $\left(28^{\circ} 28^{\prime} S\right.$, 1134ㄱ'E), M. Liddell et al., 20.04.1974; WAM C 11919 (1 male), Easter Group, coll. Sixth Abrolhos Expedition, 31.08.1972; WAM C 11929 (1 male), N of Leo I. (28 $\left.41^{\prime} \mathrm{S}, 113^{\circ} 51^{\prime} \mathrm{E}\right), 1.0 \mathrm{~m}$, coll. Sixth Abrolhos Expedition, 31.08.1972; WAM C 15775 (3 males, 2 females), Rottnest I., C. Vlamingh $\left(32^{\circ} 01^{\prime} \mathrm{S}, 115^{\circ} 30^{\prime} \mathrm{E}\right)$, on $P$. septata, coll. E. Hodgkin, -.11.1958; WAM C 24501 (1 male, 1 female), Rottnest I., Salmon Bay (32 $\left.01.2^{\prime} \mathrm{S}, 115^{\circ} 31.1^{\prime} \mathrm{E}\right), 2.0$ $\mathrm{m}$, on $P$. damicornis, coll. $\mathrm{P}$. Castro and $\mathrm{M}$. Titelius, 03.02.2000. Cocos (Keeling) Islands, Indian Ocean. WAM C 19553, (1 male), stn 7, NW of Horsburgh I., to $18 \mathrm{~m}$, coll. G. Morgan, 10.02.1989; WAM C 19787 (1 male, 2 females), stn 22, SE of Horsburgh I., to $35 \mathrm{~m}$, coll. G. Morgan, 20.02.1989; WAM C 19954 (1 male, 2 females), stn 27, Home I., coll. L. Marsh, 21.02.1989; WAM C 19473 ( 2 males, 2 females), stn 12, S end of West I., coll. G. Morgan, 19.02.1989.

\section{Trapezia serenei Odinetz, 1984}

\section{Western Australian records}

Berry and Morgan, 1986: 61 (Scott Reef, as 
Trapezia cf. ferruginea); Jones and Morgan, 1994: 178 (colour, as Trapezia ferruginea); 2002: 178 (colour); Castro, 1997a: 92 (Dampier Archipelago); Davie, 2002: 501, 502 (NW coast).

\section{Material examined}

Western Australia. QM W 17861 (1 juv. male), Hibernia Reef, reef slope, $\left(11^{\circ} 59.2^{\prime} \mathrm{S}, 123^{\circ} 22.9^{\prime} \mathrm{E}\right)$, 12.0 m, coll. J. Short, 13.05.1992; QM W 17599 (1 male, 1 female), QM W 17602 (1 juv. female), Cartier Reef, reef slope, $\left(12^{\circ} 32.2^{\prime} \mathrm{S}, 123^{\circ} 33.4^{\prime} \mathrm{E}\right), 13.0$ $\mathrm{m}$, coll. J. Short, 06.05.1992; WAM C 24482 (1 female), Kimberley region, Cassini I. (13 ${ }^{\circ} 56^{\prime} \mathrm{S}$, $\left.125^{\circ} 37^{\prime} \mathrm{E}\right), 4.0-24.0 \mathrm{~m}$, coll. KIRE 1991, 1718.08.1991; WAM C 27503 (1 male, 1 female), stn DA4/00/14, Dampier Arch., NW of Legendre I. $\left(20^{\circ} 21.206^{\prime} \mathrm{S}, 116^{\circ} 50.439^{\prime} \mathrm{E}\right), 9.0-10.0 \mathrm{~m}$, on Pocillopora verrucosa, coll. P. Castro, 30.07.2000; WAM C 22673 ( 2 males, 2 females), Kendrew I. $\left(20^{\circ} 28.5^{\prime} \mathrm{S}, 116^{\circ} 32^{\prime} \mathrm{E}\right), 6.0 \mathrm{~m}, 20.05 .1974$, coll. Crown-of-Thorns Survey. Cocos (Keeling) Islands, Indian Ocean. WAM C 19537 (1 male, 1 female), stn 7, NW of Horsburgh I., to $18 \mathrm{~m}$, coll. G. Morgan, 10.02.1989.

\section{Remarks}

Although Castro (1999a) does not list it among the Indian Ocean trapeziids, it had been previously recorded for Western Australia (Castro 1997a). A juvenile specimen from Scott Reef originally identified as Trapezia of. ferruginea by Berry and Morgan (1986: 61; WAM C 14139) could not be identified with certainty but was possibly $T$. serenei.

\section{Trapezia tigrina Eydoux and Souleyet, 1842}

\section{Western Australian records \\ Davie, 2002: 502 (NW coast).}

\section{Material examined \\ Western Australia. QM W 17595 (1 male, 1 female), Cartier Reef, reef slope, $\left(12^{\circ} 32.2^{\prime} \mathrm{S}\right.$, $\left.123^{\circ} 33.4^{\prime} \mathrm{E}\right), 13.0 \mathrm{~m}$, coll. J. Short, 6.05.1992; WAM C 22461 (1 male), stn 8, NW point of South Muiron I. $\left(21^{\circ} 39.45^{\prime} \mathrm{S}, 114^{\circ} 20.72^{\prime} \mathrm{E}\right), 11.0-13.0 \mathrm{~m}$, on Pocillopora eydouxi, coll. M. Titelius et al., 22.08.1995.}

\section{Remarks}

This species is widely distributed throughout the Indo-west Pacific region (Castro 1999a, 2000). In Australia it has only been recorded from Western Australia (Davie, 2002).
Family Tetraliidae Castro, Ng and Ahyong, 2004

Tetralia Dana, 1851

\section{Tetralia cinctipes Paul'son, 1875}

Western Australian records

Jones and Morgan, 1994: 179 (colour, as Tetralia glaberrima); 2002: 179 (colour); Davie, 2002: 495 (NW coast).

\section{Material examined}

Western Australia. WAM C 24492 (2 males, 2 females), Rowley Shoals $\left(17^{\circ} 30^{\prime} \mathrm{S}, 119^{\circ} 00^{\prime} \mathrm{E}\right)$, coll. Rowley Shoals Expedition 21.07.1986; WAM C 27496 (1 male, 1 female), Dampier Arch., NW of Legendre I. $\left(20^{\circ} 21.206^{\prime} \mathrm{S}, 116^{\circ} 50.439^{\prime} \mathrm{E}\right)$, stn DA4/ 00/14, 9.0-10.0 m, on Acropora sp., coll. P. Castro, 30.07.2000; WAM C 22462 ( 1 male, 1 female), stn 2, NE of South Muiron I. (21 $\left.{ }^{\circ} 40.46^{\prime} \mathrm{S}, 114^{\circ} 20.91^{\prime} \mathrm{E}\right)$, 3.0-5.0 m, col. M. Titelius et al.,15.08.1995; AM P 45106 (2 males, 2 females), Ningaloo, $\mathrm{W}$ of $\mathrm{Pt}$ Cloates $\left(22^{\circ} 42^{\prime} \mathrm{S}, 113^{\circ} 39^{\prime} \mathrm{E}\right), 1.0 \mathrm{~m}$, coll. Ningaloo Expedition, 30.08.1968. Christmas Island, Indian Ocean. WAM C 24093 (1 female), Dolly Beach, -.02.1987, coll. S. Slack-Smith; WAM C 24494 (1 male), stn 11, West White Beach, 5.0-30.0 m, 18.02.1987.

\section{Remarks}

Known throughout the Indo-west Pacific region (Castro 1999a; 2000). Live specimens from the Dampier Archipelago (WAM C 27496) showed the characteristic blue band across the frontal border of the carapace observed in other locations (see Jones and Morgan 1994: 179; as T. glaberrima).

\section{Tetralia glaberrima (Herbst, 1790)}

Tetralia glaberrima fulva Serène, 1984: 282.

Tetralia glaberrima - Castro et al., 2004: 25, 26, fig. 3, pls 1D, 1E, $2 \mathrm{~A}$.

\section{Western Australian records}

Berry and Morgan, 1986: 61 (Scott Reef, as Tetralia glaberrima fulva); Castro, 1999a: 101 (Christmas I., as Tetralia fulva); Davie, 2002: 496 (NW coast, as Tetralia fulva).

\section{Material examined}

Western Australia. WAM C 20580 (1 male, 1 female), KIRE $1991 \mathrm{stn} 15$, Kimberley region, $\mathrm{W}$ of Cassini I. (13 $\left.{ }^{\circ} 56^{\prime} \mathrm{S}, 125^{\circ} 37^{\prime} \mathrm{E}\right), 24.0-25.0 \mathrm{~m}, 17-$ 18.08.1991; WAM C 2494 (1 male), Scott Reef $\left(14^{\circ} 03^{\prime} \mathrm{S}, 121^{\circ} 46^{\prime} \mathrm{E}\right)$, coll. P. Berry, 7.09.1984; WAM C 20586 ( 1 male, 1 female), KIRE 1991 stn 27, Rob Roy Reef $\left(14^{\circ} 25^{\prime} \mathrm{S}, 124^{\circ} 51^{\prime} \mathrm{E}\right), 3.0-22.0 \mathrm{~m}$, 21.08.1991; QM W20125 (1 male), stn 3, S of Sunday I. (16 $\left.25.4^{\prime} \mathrm{S}, 123^{\circ} 09^{\prime} \mathrm{E}\right), 12.0 \mathrm{~m}, 16.11 .1994$; 
coll. J. Short, WAM C 27497 (2 males, 1 female), stn DA4/00/14, Dampier Arch., NW of Legendre I. $\left(20^{\circ} 21.206^{\prime} \mathrm{S}, 116^{\circ} 50.439^{\prime} \mathrm{E}\right), 9.0-10.0 \mathrm{~m}$, on Acropora sp., coll. P. Castro, 30.07.2000; WAM C 24472 (1 male, 1 female), Kendrew I., reef flat (20²9'S, $\left.116^{\circ} 32^{\prime} \mathrm{E}\right)$, coll. L. Marsh and R. George, 11.10.1972; WAM C 27512 (1 male, 1 female), stn DA4/00/01, W of Angel I. (20²9.05'S, 116 $\left.47.83^{\prime} \mathrm{E}\right)$, $4.0 \mathrm{~m}$, on Acropora sp., coll. P. Castro, 25.07.2000; WAM C 22463 (1 female), stn 12, SE of South Muiron I. (21'39.19'S, $\left.114^{\circ} 22.47^{\prime} \mathrm{E}\right), 6.0-13.0 \mathrm{~m}$, coll. M. Titelius et al., 24.08.1995. Christmas Island, Indian Ocean. WAM C 23890 (1 female), stn 12, Egeria Pt., coll. G. Morgan, 19.02.1987. Cocos (Keeling) Islands, Indian Ocean. WAM C 19896 (1 male), stn 27, Home I., outer reef, coll. P. Berry, 21.02.1989.

\section{Remarks}

It is possible that some of the preserved specimens identified as $T$. glaberrima may belong to a colour variety (Tetralia aff. glaberrima, see below) identifiable only by its colour pattern. The colour has been unfortunately lost after at least two years of preservation.

\section{Tetralia aff. glaberrima (Herbst, 1790)}

\section{Material examined}

Western Australia. WAM C 27517 (1 male, 1 female), stn DA3/99/67, Dampier Arch., Nelson Rocks $\left(20^{\circ} 26.51^{\prime} \mathrm{S}, 116^{\circ} 40.26^{\prime} \mathrm{E}\right)$, coll. M. Titelius et al., 07.09.1999; WAM C 27518 (1 male), stn DA3/99/ 47, Kendrew I. $\left(20^{\circ} 28.94^{\prime} \mathrm{S}, 116^{\circ} 32.52^{\prime} \mathrm{E}\right), 4.0-5.0 \mathrm{~m}$, coll. M. Titelius et al. 30.08.1999; WAM C 27516 (1 male), stn DA4/00/24, SW end; Enderby I. (2037.10'S, $\left.116^{\circ} 26.72^{\prime} \mathrm{E}\right), 16.0 \mathrm{~m}$, on Acropora sp., coll. P. Castro, 03.08.2000.

\section{Remarks}

Four specimens from the Dampier Archipelago were morphologically identical to $T$. glaberrima (= T. fulva) except for a different colour pattern. The walking legs had red spots along the anterior portion of the carpi (distal edge), propodi, and dactyli (proximal edge). The carapace and chelipeds of the only specimen that was observed alive (male, WAM C 27516) were of the same light orange brown colour as in $T$. glaberrima but clearly lighter in colour (light orange brown) than in T. glaberrima

\section{Tetralia nigrolineata Serène and Dat, 1957}

\section{Western Australian records}

Morgan, 1990: 51 (Kimberley region, as Tetralia glaberrima obscura); Davie, 2002: 496 (NW coast).

\section{Material examined}

Western Australia. WAM C 20737 (1 male, 1 female), stn 14, Kimberley region, Long Reef, intertidal $\left(13^{\circ} 48^{\prime} \mathrm{S}, 125^{\circ} 47^{\prime} \mathrm{E}\right)$, coll. KIRE 1991, 17.08.1991; WAM C 20533 (2 males, 2 females), stn 15, Kimberley region, Long Reef, $12.0 \mathrm{~m}$, coll. KIRE 1991, 17.08.1991; WAM C 20750 (1 male), stn 9, Sir Graham Moore Is., Scorpion I., intertidal $\left(13^{\circ} 52^{\prime} \mathrm{S}\right.$, 126³6'E), coll. KIRE 1991, 14-15.08.1991; WAM C 20579 (2 males), stn 15, W of Cassini I. (13 ${ }^{\circ} 56^{\prime} \mathrm{S}$, $\left.125^{\circ} 37^{\prime} \mathrm{E}\right), 24.0-25.0 \mathrm{~m}$, coll. KIRE 1991, 1718.08.1991; WAM C 20654 (1 male, 1 female), stn 4, Tranquil Bay $\left(13^{\circ} 56^{\prime} \mathrm{S}, 1^{\circ} 7^{\circ} 18^{\prime} \mathrm{E}\right)$, coll. KIRE 1991, 13.08.1991; QM W 21189 (1 male, 1 female), stn 9, Long I., Vansittart Bay (1358.9'S, 126 19.6'E), coll. J. Short, 24.11.1995; WAM C 20558 (5 males, 4 females), stn 20, Solem I., intertidal (14\%09'S, 12539'E), coll. KIRE 1991, 19.08.1991; WAM C 20669 ( 1 male, 1 female), stn 21, Solem I., intertidal (1409'S, 125 $\left.{ }^{\circ} 39^{\prime} \mathrm{E}\right)$, coll. KIRE 1991, 19.08.1991; WAM C 20578 (1 male, 1 female), stn 24, Albert Reef (14'15'S, $\left.125^{\circ} 09^{\prime} \mathrm{E}\right), 3.0-15.0 \mathrm{~m}, 20.08 .1991$; coll. KIRE 1991; WAM C 18823 (1 male, 1 female), stn 50, Don I. $\left(14^{\circ} 16^{\prime} \mathrm{S}, 125^{\circ} 19^{\prime} \mathrm{E}\right), 8.0 \mathrm{~m}$, coll. G. Morgan, 16.07.1988; WAM C 20553 (1 male, 2 females), sth 23. Hale I. $\left(14^{\circ} 22^{\prime} \mathrm{S}, 125^{\circ} 25^{\prime} \mathrm{E}\right), 3.0-11.0 \mathrm{~m}$, 20.08.1991, KIRE 1991; WAM C 20587 (2 males, 2 females), stn 27 , Rob Roy Reef (14 $\left.25^{\prime} \mathrm{S}, 124^{\circ} 51^{\prime} \mathrm{E}\right)$, coll. KIRE 1991, 21.08.1991; WAM C 18910 (5 males, 5 females), Rob Roy Reef (14²5.6'S, 124 51.9'E), stn 49, coll. G. Morgan, 15.07.1988; WAM C 22666 (1 male, 1 female), Rob Roy Reef, on Acropora valida, coll. M. Titelius et al., 26.11.1996; WAM C 22667 (1 male), intertidal, Maret I. (14'25.6'S, $\left.124^{\circ} 58.9^{\prime} \mathrm{E}\right)$, coll. M. Titelius et al., 27.11.1996; WAM C 22665 (1 male), Jesseux I. $\left(14^{\circ} 42.77^{\prime} \mathrm{S}, 124^{\circ} 58.91^{\prime} \mathrm{E}\right), \operatorname{stn} 17$, 7.0-32.0 m, coll. M. Titelius et al. 26.11.1996; WAM C 19118 (1 female), stn 85, W of Buffon I. (14 ${ }^{\circ} 55^{\prime}$ 'S, $\left.124^{\circ} 44^{\prime} \mathrm{E}\right), 3.0-22.0 \mathrm{~m}$, G. Morgan, 23.07.1988; WAM C 19242 ( 1 male, 1 female), stn 101 , Lucas I. (15¹3'S, $\left.124^{\circ} 29^{\prime} \mathrm{E}\right), 3.0-29.0 \mathrm{~m}$, coll. G. Morgan, 24.07.1988; WAM C 21844 ( 2 males, 1 female), Monte Bello Is., $\mathrm{W}$ of Alpha I. $\left(20^{\circ} 24.5^{\prime} \mathrm{S}, 115^{\circ} 30^{\prime} \mathrm{E}\right), 3.0-4.0 \mathrm{~m}$, coll. L. Marsh et al., 12.08.1993; WAM C 24491 (1 female), $W$ of Hermite I. $\left(20^{\circ} 27^{\prime} S, 115^{\circ} 29^{\prime} \mathrm{E}\right), 6.0-7.0$ $\mathrm{m}$, coll. L. Marsh et al., 10.08.1993; WAM C 21834 (1 female), W of Hermite I., SW of barrier reef, intertidal reef flat, $\left(20^{\circ} 30^{\prime} \mathrm{S}, 115^{\circ} 27^{\prime} \mathrm{E}\right)$, L. Marsh et al., 19.08.1993; WAM C 27513 (1 male, 1 female, 1 juv. male), stn DA4/00/14, Dampier Arch., NW of Legendre I. (20²1.206'S, 116 $\left.50.439^{\prime} \mathrm{E}\right), 9.0-10.0 \mathrm{~m}$, on Acropora sp., coll. P. Castro, 30.07.2000; WAM C 27506 ( 1 female), Legendre I., $\left(20^{\circ} 21.270^{\prime} \mathrm{S}\right.$, $\left.116^{\circ} 50.557^{\prime} \mathrm{E}\right)$, stn DA4/00/37, $7.5 \mathrm{~m}$, on Acropora sp., coll. P. Castro and C. Bryce, 06.08.2000; WAM C 25301 (1 male), WAM C 25508 (1 male), Legendre I. $\left(20^{\circ} 24.32^{\prime} \mathrm{S}, 116^{\circ} 56.11^{\prime} \mathrm{E}\right)$, stn DA1/98/03, M. Titelius et al., 18.10.1998; WAM C28107 (1 female), stn DA3/ 99/40, Brigadier I. $\left(20^{\circ} 26.657^{\prime} \mathrm{S}, 116^{\circ} 36.507^{\prime} \mathrm{E}\right), 6.0$ 
$14.0 \mathrm{~m}$, coll. M. Titelius et al., 28.08.1999, WAM C 27499 ( 1 male, 1 female), W of Gidley I. (20²7.695'S, $\left.116^{\circ} 47.512^{\prime} \mathrm{E}\right)$, stn DA4/00/34, $10 \mathrm{~m}$, on Acropora sp., coll. P. Castro and C. Bryce, 05.08.2000; WAM C 15983 (3 males, 3 females), Kendrew I. (20²8.5'S, $\left.116^{\circ} 32^{\prime} \mathrm{E}\right)$, coll. Crown-of-Thorns Survey, 06.05.1973; WAM C 11885 (1 female), Kendrew I. (20²8.5'S, $\left.116^{\circ} 32^{\prime} \mathrm{E}\right)$, coll. Crown-of-Thorns Survey, 10.05.1973; WAM C 16032 (1 female), Kendrew I. (20²8.5'S, $\left.116^{\circ} 32^{\prime} \mathrm{E}\right)$, coll. Crown-of-Thorns Survey, 10.10.1972; WAM C 14316 (1 male), Kendrew I. (20²8.5'S, $\left.116^{\circ} 32^{\prime} \mathrm{E}\right)$, coll. Crown-of-Thorns Survey, 01.11.197; WAM C 24488 (1 male), WAM C 16018 (1 female), Kendrew I. $\left(20^{\circ} 28.5^{\prime} \mathrm{S}, 116^{\circ} 32^{\prime} \mathrm{E}\right)$, coll. Crown-ofThorns Survey, 02.11.1974; WAM C 27493 (2 males, 2 females), stn DA4/00/01, W of Angel I. (20²9.05'S, $\left.116^{\circ} 47.83^{\prime} \mathrm{E}\right), 4.0 \mathrm{~m}$, on Acropora sp., coll. P. Castro, 25.07.2000; WAM C 25382 (1 male), stn DA1/98/08, Angel I. (20²9.18'S, $\left.116^{\circ} 47.71 ' \mathrm{E}\right)$, M. Titelius et al., 20.10.1998; WAM C 25394 (2 males), DA1/98/08, Angel I. (20²9.18'S, $\left.116^{\circ} 47.71^{\prime} \mathrm{E}\right)$, M. Titelius et al., 20.10.1998; WAM C 25385 ( 1 male, 1 female), DA1/ 98/08, Angel I. (20²9.18'S, $\left.116^{\circ} 47.71^{\prime} \mathrm{E}\right), \mathrm{M}$. Titelius et al., 20.10.1998; WAM C 25386 (1 female), DA1/98/ 08, Angel I. (20²9.18'S, $\left.116^{\circ} 47.71^{\prime} \mathrm{E}\right), \mathrm{M}$. Titelius et al., 20.10.1998; WAM C 25390 (1 female), DA1/98/ 08, Angel I. (20²9.18'S, $\left.116^{\circ} 47.71^{\prime} \mathrm{E}\right), \mathrm{M}$. Titelius et al., 20.10.1998; WAM C28108 (1 male, 1 female), Kendrew I. (20²9.671'S, $\left.116^{\circ} 35.894^{\prime} \mathrm{E}\right)$, stn DA3/99/ 46, 3.0-11.0 m, coll. M. Titelius et al., 30.08.1999; WAM C 27504 (7 males, 7 females), stn DA4/00/32, $\mathrm{W}$ of Angel I. $\left(20^{\circ} 29.765^{\prime} \mathrm{S}, 116^{\circ} 47.480^{\prime} \mathrm{E}\right), 8.0 \mathrm{~m}$, on Acropora sp., coll. P. Castro, 04.08.2000; WAM C 27509 (4 males, 3 females), NW corner of Roly Rock (20⒉88'S, $\left.116^{\circ} 30.05^{\prime} \mathrm{E}\right)$, stn DA4/00/23, $15.0 \mathrm{~m}$, on Acropora sp., coll. P. Castro, 02.08.2000; WAM C 28109 (1 female), stn DA3/99/36, Malus Is. (2030.050'S, $\left.116^{\circ} 40.594^{\prime} \mathrm{E}\right), 6.0-14.0 \mathrm{~m}$, on Pocillopora damicornis, coll. M. Titelius et al., 27.08.1999; WAM C28110 (1 male), stn DA3/99/37, Malus Is., (20³0.632'S, $\left.116^{\circ} 38.788^{\prime} \mathrm{E}\right), 2.3-5.0 \mathrm{~m}, \mathrm{M}$. Titelius et al., 27.08.1999; WAM C 28110 (1 male), stn DA3/99/48, Goodwin I., intertidal, (20³2'S, 116³2'E), M. Titelius et al., 31.08.1999; WAM C 28112 (1 female), stn DA3/99/58, Enderby I. (2034.398'S, $\left.116^{\circ} 33.443^{\prime} \mathrm{E}\right), 2.5-4.0 \mathrm{~m}$, coll. M. Titelius et al., 03.09.1999; WAM C 28113 (1 female), stn DA3/99/53, Enderby I. (20³4'52'S, 116 $\left.34^{\prime} 58^{\prime} \mathrm{E}\right)$, coll. M. Titelius et al., 01.09.1999; WAM C 27488 (1 male, 1 female), stn DA4/00/10, W of Enderby I. (20³5.385'S, $\left.116^{\circ} 28.572 ' \mathrm{E}\right), 3.0 \mathrm{~m}$, on Acropora sp., coll. P. Castro, 28.07.2000; WAM C 27505 (5 males, 8 females), stn DA4/99/04, SW tip of West Lewis I. (20³6.16'S, $\left.116^{\circ} 35.74^{\prime} \mathrm{E}\right), 2.0-3.0 \mathrm{~m}$, on Acropora sp., coll. P. Castro, 26.07.2000; WAM C 27502 (3 males, 4 females), stn DA4/00/08, SW tip of West Lewis I. $\left(20^{\circ} 36.310^{\prime} \mathrm{S}, 116^{\circ} 35.696^{\prime} \mathrm{E}\right), 5.0 \mathrm{~m}$, on Acropora sp., coll. P. Castro, 27.07.2000; WAM C 22468 (1 male, 1 female), stn 13, SE of South Muiron.
I. (2139.13'S, $\left.114^{\circ} 22.26^{\prime} \mathrm{E}\right), 1.0-2.0 \mathrm{~m}$, coll. M. Titelius et al., 24.08.1995; WAM C 22474 (1 female), WAM C 22476 (1 female), stn 2 , NE of South Muiron I. (21 $\left.{ }^{\circ} 40.46^{\prime} \mathrm{S}, 114^{\circ} 20.91^{\prime} \mathrm{E}\right), 3.0-5.0 \mathrm{~m}$, coll. M. Titelius et al., 15.08.1995; WAM C 22475 (1 female), stn 7, E of South Muiron I. (21\% $40.60^{\prime} S$, $\left.114^{\circ} 20.75^{\prime} \mathrm{E}\right), 1.0-1.5 \mathrm{~m}$, coll. S. Slack-Smith et al., 22.08.1995; AM P 45108 (2 males, 2 females), W of Frazer I. near Pt Cloates (22 $\left.2^{\circ} 38^{\prime} S, 113^{\circ} 37.5^{\prime} \mathrm{E}\right)$, 29.08.1968; AM P 45105 (2 males, 2 females), Ningaloo, W of Pt Cloates (22 $\left.{ }^{\circ} 42^{\prime} \mathrm{S}, 113^{\circ} 39^{\prime} \mathrm{E}\right), 1.0 \mathrm{~m}$, coll. Ningaloo Expedition, 28.08.1968; WAM C 22473 (1 male), stn 26, Shark Bay, $N$ end of Bernier I. $\left(24^{\circ} 45.54^{\prime} \mathrm{S}, 113^{\circ} 09.65^{\prime} \mathrm{E}\right), 0.5-4.0 \mathrm{~m}, 20.05 .1995$; WAM C 22455 (1 female), NE of Bernier I.

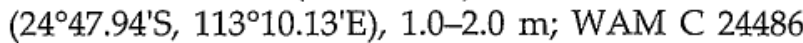
(1 male), stn 1, Shark Bay, NE of Dorre I. (24 $4^{\circ} 5.7^{\prime} \mathrm{S}$, $\left.113^{\circ} 07.3^{\prime} \mathrm{E}\right)$, coll. M. Titelius et al., 11.05.1995; WAM C 15987 (1 female), W of Kelvin Right anchorage $\left(26^{\circ} 10.20^{\prime} \mathrm{S}, 113^{\circ} 11^{\prime} \mathrm{E}\right), 2.0 \mathrm{~m}$, coll. N. Sarti, 11.04.1979; WAM C 24499 (1 male, 1 female), Rottnest I., Salmon Bay ( $\left.32^{\circ} 01.2^{\prime} S, 115^{\circ} 31.1^{\prime} \mathrm{E}\right), 2.0$ $\mathrm{m}$, coll. P. Castro and M. Titelius, 03.02.2000.

\section{Remarks}

Live specimens of $T$. nigrolineata collected in Western Australia showed a wide variation of colour forms, varying from light to dark brown, a situation already observed elsewhere (see Castro, 1997a). Smaller individuals tended to be lighter in colour. It is by far the most common symbiotic crab of Acropora in inshore locations.

\section{Tetralia rubridactyla Garth, 1971}

\section{Western Australian records \\ Davie, 2002: 496 (NW coast).}

\section{Material examined}

Western Australia. QM W 17598 (1 male, 1 female), Cartier Reef, reef slope, $\left(12^{\circ} 32.2^{\prime} \mathrm{S}\right.$, $\left.123^{\circ} 33.4^{\prime} \mathrm{E}\right), 13.0 \mathrm{~m}$, coll. J. Short, 6.05.1992; WAM C 22942 (1 male), stn 18, Kimberley region, Colbert I., SE reef $\left(14^{\circ} 52.6^{\prime} \mathrm{S}, 124^{\circ} 43.0^{\prime} \mathrm{E}\right)$, coll. M. Titelius et al., 01.12.1996; WAM C 27500 (3 males, 4 females), Dampier Arch., NW of Legendre I. $\left(20^{\circ} 21.206^{\prime} \mathrm{S}\right.$, $\left.116^{\circ} 50.439^{\prime} \mathrm{E}\right)$, stn DA4/00/14, 9.0-10 m, on Acropora sp., coll. P. Castro, 30.07.2000; WAM C 27511 (1 male, 1 female), stn DA4/00/16, NW of Legendre I. (20²4.025'S, $\left.116^{\circ} 55.079^{\prime} \mathrm{E}\right), 13.0-14.0 \mathrm{~m}$, on Acropora sp., coll. P. Castro, 31.07.2000; WAM C 25304 (1 male), WAM C 25497 (1 female), stn DA1/ 98/03, Legendre I. (20²4.32'S, 116 $56.11 ' \mathrm{E}), \mathrm{M}$. Titelius et al., 18.10.1998; WAM C 28114 (1 male), stn DA3/99/40, Brigadier I. $\left(20^{\circ} 26.657^{\prime} \mathrm{S}\right.$, $\left.116^{\circ} 36.507^{\prime} \mathrm{E}\right), 6.0-14.0 \mathrm{~m}$, coll. M. Titelius et al., 28.08.1999; WAM C 27489 (1 male, 1 female), stn DA4/00/34, W of Gidley I. (20²7.695'S, 
$\left.116^{\circ} 47.512^{\prime} \mathrm{E}\right), 10.0 \mathrm{~m}$, on Acropora sp., coll. P. Castro and C. Bryce, 05.08.2000; WAM C 16019 (1 male, 1 female), WAM C 15939 ( 1 male, 1 female), Kendrew I. $\left(20^{\circ} 29^{\prime} \mathrm{S}, 116^{\circ} 32^{\prime} \mathrm{E}\right), 08.05 .1973$, coll. Crown-of-Thorns Survey, 16.10.1973; WAM C 22480 (1 male, 1 female), stn 13, South Muiron I. (213․ $\left.13^{\prime} \mathrm{S}, 114^{\circ} 22.26^{\prime} \mathrm{E}\right), 1.0-2.0 \mathrm{~m}$, coll. M. Titelius et al., 24.08.1995; WAM C 22477 ( 1 male, 1 female), stn 13, SE of South Muiron I. (21'39.13'S, $\left.114^{\circ} 22.26^{\prime} \mathrm{E}\right), 1.0-2.0 \mathrm{~m}$, coll. M. Titelius et al., 24.08.1995; AM P 45107 ( 2 males, 2 females), Ningaloo, $\mathrm{W}$ of Pt Cloates $\left(22^{\circ} 42^{\prime} \mathrm{S}, 113^{\circ} 39^{\prime} \mathrm{E}\right), 1.0 \mathrm{~m}$, coll. Ningaloo Expedition, 30.08.1968; WAM C 24489 (1 male), stn 2, Shark Bay, NE of Dorre I., off Quoin Bluff $\left(25^{\circ} 03.3^{\prime} \mathrm{S}, 113^{\circ} 07.5^{\prime} \mathrm{E}\right)$, coll. M. Titelius et al., 12.05.1995; WAM C 22478 (1 male, 1 female), stn 13, Dorre I., $W$ coast $N$ of Boulder Cliff $\left(25^{\circ} 09.04^{\prime} \mathrm{S}, 113^{\circ} 05.33^{\prime} \mathrm{E}\right), 5.0-22.0 \mathrm{~m}$, coll. S. SlackSmith, 16.05.1995; WAM C 11736 (1 male, 1 female), Houtman Abrolhos Is., Beacon I. $\left(28^{\circ} 28^{\prime} \mathrm{S}, 113^{\circ} 47^{\prime} \mathrm{E}\right)$, coll. S. Bingham, 21.04.1974; WAM C 11930 (1 male), stn D23, Wooded I. (28 $\left.45^{\prime} \mathrm{S}, 113^{\circ} 48^{\prime} \mathrm{E}\right), 1.0 \mathrm{~m}$, coll. M. Liddell, 01.09.1972; WAM C 16030 (3 males, 2 females), Stick I. ( $\left.28^{\circ} 53^{\prime} \mathrm{S}, 113^{\circ} 55^{\prime} \mathrm{E}\right)$, coll. A. James and G. Davenport, 07.01.1968; WAM C 24500 (1 male), Rottnest I., Salmon Bay $\left(32^{\circ} 01.2^{\prime} \mathrm{S}\right.$, $\left.115^{\circ} 31.1^{\prime} \mathrm{E}\right), 2.0 \mathrm{~m}$, coll. P. Castro and M. Titelius, 03.02.2000. Christmas Island, Indian Ocean. WAM C 23892 (2 males, I female), West White Beach, 5.0$30.0 \mathrm{~m}, 18.02 .1987$.

\section{Remarks}

The colour pattern of live individuals was similar to that observed among Coral Sea and western Pacific populations (see Castro 1999b). The fingers, however, were closer to those of western Indian Ocean populations: orange to light brown instead of the orange-red to red-brown dorsal portion of the dactylus (particularly of the largest cheliped) in the western Pacific populations (see Castro 1997a: pl. 1, fig. E). The species is widely distributed throughout the Indo-west Pacific region. In Australia it is known from Queensland (Great Barrier Reef, NE coast) and Western Australia (NW coast) (Davie, 2002).

\section{Tetralia muta (Linnaeus, 1758)}

Tetralia vanninii Galil and Clark, 1988: 146, figs 1C, $2 \mathrm{~B}, 3 \mathrm{C}, 4 \mathrm{C}, 4 \mathrm{H}, 6 \mathrm{C}$.

Tetralia muta - Castro et al., 2004: 25, 29.

\section{Western Australian records}

Davie, 2002: 496 (NW coast, as Tetralia vanninii).

\author{
Material examined \\ Western Australia. WAM C 25398 (1 female), stn \\ DA1/98/08, Dampier Arch., Angel I. $\left(20^{\circ} 29.18^{\prime} \mathrm{S}\right.$,
}

$\left.116^{\circ} 47.71^{\prime} \mathrm{E}\right), \mathrm{M}$. Titelius et al., 20.10.1998; WAM C 24487 ( 1 female), Houtman Abrolhos Is., Coronation I. $\left(28^{\circ} 52^{\prime} S, 113^{\circ} 59^{\prime} \mathrm{E}\right)$, coll. G. Morgan, -.10.1985; WAM C 24498 (1 male), Rottnest I., Salmon Bay ( $\left.32^{\circ} 01.2^{\prime} \mathrm{S}, 115^{\circ} 31.1^{\prime} \mathrm{E}\right), 2.0 \mathrm{~m}$, coll. P. Castro and $\mathrm{M}$. Titelius, 03.02.2000. Christmas Island, Indian Ocean. WAM C 24092 (1 male. 1 female), off Merial Beach, 3.0-12.0 m, coll. S. Slack-Smith and L. Marsh, 17.02.1987.

\section{Remarks}

Tetralia muta is known from most of the Indowest Pacific (Castro 1999a; 2000, as T. vanninii). It has been recorded from both the east and west coasts of Australia: Great Barrier Reef, Queensland and NW coast of Western Australia (Davie, 2002).

The colour of the single specimen that was observed alive (WAM C 24498) had a black band (followed proximally by a thin blue line) along the anterior border of the white carapace, red fingers and a red tubercle on the proximal edge of each cheliped propodus. This colour pattern is similar to that observed among specimens from French Polynesia (Castro, 1997b: 114) and Guam (Castro, 2003). Colour variations were observed among Guam specimens, however. The situation is further complicated because the live colour of the type material is unknown. Preserved juveniles and small specimens of Tetralia nigrolineata where some of the colour pattern has been lost are difficult to distinguish from juveniles of $T$. muta so it is possible that specimens identified as $T$. nigrolineata belong instead to T. muta.

\section{Tetraloides Galil, 1986}

\section{Tetraloides heterodactyla (Heller, 1861)}

\section{Western Australian records}

Berry and Morgan, 1986: 61 (Scott Reef, as Tetralia heterodactyla fusca); Davie, 2002: 497 (NW coast).

\section{Material examined}

Western Australia. QM W 17786 (3 males, 2 females), Hibernia Reef, patch reef, (11 $58.8^{\prime} \mathrm{S}$, $\left.123^{\circ} 21.2^{\prime} \mathrm{E}\right), 9.0 \mathrm{~m}$, coll. R. Kelley, 11.05.1992.

\section{Tetraloides nigrifrons (Dana, 1852)}

\section{Western Australian records}

Jones and Morgan, 1994: 178 (colour, as Tetralia heterodactyla); 2002: 178 (colour); Davie, 2002: 497 (NW coast).

\author{
Material examined \\ Western Australia. QM W 17633 (1 male, 1 \\ female), reef slope, Cartier Reef (12 $32.2^{\prime} S$,
}


$\left.123^{\circ} 33.4^{\prime} \mathrm{E}\right), 13.0 \mathrm{~m}$, on gorgonacean coral, coll. J. Short, 06.05.1992; AM ex P 45105 (1 male, 1 female), Ningaloo, $W$ of Point Cloates $\left(22^{\circ} 42^{\prime} S\right.$, $\left.113^{\circ} 39^{\prime} \mathrm{E}\right), 1.0 \mathrm{~m}$, coll Ningaloo Expedition, 28.08.1968; WAM C 17418 (1 male), Houtman Abrolhos Is, Coronation I. (28 $52^{\prime}$ S, 113 $\left.59^{\prime} \mathrm{E}\right)$, coll. G. Morgan, -.10.1985.

\section{BIOGEOGRAPHY}

The geographic distribution of trapeziids and tetraliids is determined by the distribution of their obligate coral hosts. The distribution of coral crabs is of particular interest along the coast of Western Australia, which extends from areas of highly diverse coral reefs in the Timor Sea, bordering Indonesia, to low species-diversity, high-latitude communities with only scattered colonies of reefbuilding corals but no structural reefs south of the Houtman Abrolhos Islands (see Veron and Marsh,
1988; Veron, 1993). The distribution of the species of Tetralia, Tetraloides, and Trapezia is summarized in Table 1.

All seventeen species belonging to these three genera present in Western Australia are also known from Indonesia (Castro, 1999b) except two species: Tetralia muta, a widespread Indo-west Pacific species and Tetralia aff. glaberrima. Trapezia bidentata (Forskål, 1775) ( $=$ T. ferruginea Latreille, 1828) and T. rufopunctata (Herbst, 1799) are widelydistributed species known from Indonesia, the Cocos (Keeling) Islands, and Christmas Island (Castro 1999a: 109, 114) but are yet to be identified from Western Australia. The specimen of $T$. rufopunctata illustrated by Jones and Morgan (1994: 179; 2002: 179) appears not to have been collected in Western Australia.

The southernmost limit of the distribution of these species of coral crabs is much different (Table 1). Three species appear to be restricted to the

Table 1 Geographic distribution of trapeziid (Trapezia) and tetraliid (Tetralia, Tetraloides) crabs symbiotic with hermatypic corals along the coast of Western Australia. Species are arranged according to increasing southernmost limits of their distributions.

\begin{tabular}{|c|c|c|}
\hline Species & Northernmost limit & Southernmost limit \\
\hline Tetraloides heterodactyla & Timor Sea & $\begin{array}{l}\text { Timor Sea } \\
\left(11^{\circ} 58^{\prime} \mathrm{S}\right)\end{array}$ \\
\hline Trapezia plana & Timor Sea & $\begin{array}{l}\text { Timor Sea } \\
\left(12^{\circ} 30^{\prime} \mathrm{S}\right)\end{array}$ \\
\hline Trapezia guttata & Timor Sea & $\begin{array}{l}\text { Southern Kimberley region } \\
\left(15^{\circ} 21^{\prime} \mathrm{S}\right)\end{array}$ \\
\hline Trapezia serenei & Timor Sea & $\begin{array}{c}\text { Dampier Archipelago } \\
\left(20^{\circ} 28^{\prime} \mathrm{S}\right)\end{array}$ \\
\hline Trapezia formosa & Dampier Archipelago & $\begin{array}{c}\text { Dampier Archipelago } \\
\left(20^{\circ} 32^{\prime} S\right)\end{array}$ \\
\hline $\begin{array}{c}\text { Tetralia } \\
\text { aff. glaberrima }\end{array}$ & Dampier Archipelago & $\begin{array}{c}\text { Dampier Archipelago } \\
\left(20^{\circ} 37^{\prime} \mathrm{S}\right)\end{array}$ \\
\hline Tetralia glaberrima & Timor Sea & $\begin{array}{c}\text { Dampier Archipelago } \\
\left(21^{\circ} 39^{\prime} S\right)\end{array}$ \\
\hline Trapezia lutea & Timor Sea & $\begin{array}{l}\text { Dampier Archipelago } \\
\qquad\left(21^{\circ} 39^{\prime} \mathrm{S}\right)\end{array}$ \\
\hline Trapezia tigrina & Timor Sea & $\begin{array}{l}\text { Dampier Archipelago } \\
\left(21^{\circ} 39^{\prime} S\right)\end{array}$ \\
\hline Tetralia cinctipes & $\begin{array}{c}\text { Rowley Shoals } \\
\left(17^{\circ} 30^{\prime} \mathrm{S}\right)\end{array}$ & $\begin{array}{l}\text { Ningaloo } \\
\left(22^{\circ} 42^{\prime} S\right)\end{array}$ \\
\hline Trapezia digitalis & Dampier Archipelago & $\begin{array}{l}\text { Houtman Abrolhos Is. } \\
\qquad\left(28^{\circ} 30^{\prime} \mathrm{S}\right)\end{array}$ \\
\hline Tetraloides nigrifrons & Timor Sea & $\begin{array}{l}\text { Houtman Abrolhos Is. } \\
\qquad\left(28^{\circ} 52^{\prime} \mathrm{S}\right)\end{array}$ \\
\hline Tetralia nigrolineata & Timor Sea & $\begin{array}{c}\text { Rottnest I. } \\
\left(32^{\circ} 01^{\prime} S\right)\end{array}$ \\
\hline Tetralia rubridactyla & Timor Sea & $\begin{array}{l}\text { Rottnest I. } \\
\left(32^{\circ} 01^{\prime} S\right)\end{array}$ \\
\hline Trapezia cymodoce & Timor Sea & $\begin{array}{l}\text { Rottnest I. } \\
\left(32^{\circ} 01^{\prime} S\right)\end{array}$ \\
\hline Trapezia septata & Timor Sea & $\begin{array}{l}\text { Rottnest I. } \\
\left(32^{\circ} 01^{\prime} S\right)\end{array}$ \\
\hline Tetralia muta & Dampier Archipelago & $\begin{array}{l}\text { Rottnest I. } \\
\left(32^{\circ} 01^{\prime} S\right)\end{array}$ \\
\hline
\end{tabular}


Timor Sea and its southern limits, six as far south as the Dampier Archipelago ( $\left.20^{\circ} 28^{\prime} \mathrm{S}\right)$, one to Ningaloo $\left(22^{\circ} 42^{\prime} \mathrm{S}\right)$ and two to the Houtman Abrolhos Islands further south $\left(28^{\circ} 30^{\prime} \mathrm{S}\right)$. Five species $(29 \%$ of all species), however, are now known to extend as far as the southernmost limit of Pocillopora and Acropora at Rottnest Island $\left(32^{\circ} 01^{\prime} \mathrm{S}\right)$ off Perth. The sharp latitudinal decrease in the hosts' species diversity (from five species of Pocillopora and 48 of Acropora off the Western Australia coast of the Timor Sea to one species of Pocillopora and two of Acropora south of Perth; Veron, 1993) is, therefore, not directly correlated to the species diversity of coral crabs. There is no evidence of host specificity in the settlement of megalopa larvae among trapeziids and tetraliids, so that coral crabs are found in reefs as long as larvae are found in the plankton and colonies of Pocillopora and/or Acropora are present in the substrate.

\section{ACKNOWLEDGEMENTS}

Peter Castro thanks D.S. Jones (WAM), P. Berents (AM), P.F. Davie (QM), and J.W. Short (QM) for their generous and warm hospitalities during visits to their respective museums.

Melissa Titelius thanks her fellow expedition members, S. Morrison, C. Bryce, S. Slack-Smith, J. Fromont and B. Hutchins, for their companionship and help during the long field expeditions. $M$. Salotti, A. Anderson and R. Anderson are thanked for their help in the laboratory and data basing. Many thanks go to J. Ralston and the crew of the Kimberley Quest (Expedition DA1/98), T. Berden and the crew of the Fisheries Research Vessel Flinders (Expedition DA2/99) and B. Kirkwood and the crew on Top Gun (Expedition DA3/99), for their assistance and service during the respective expeditions.

The authors thank Diana Jones for providing the opportunity to work on this collection and Woodside Energy Ltd for providing the funding for the field component of this project and this publication.

\section{REFERENCES}

Alcock, A. (1898). Materials for a Carcinological Fauna of India. No. 3. The Brachyura Cyclometopa. Part I. The Family Xanthidae. Journal of the Asiatic Society of Bengal 67: 67-233.

Balss, H. (1935). Brachyura of the Hamburg Museum Expedition to South-Western Australia, 1905. Journal of the Royal Society of Western Australia 21: 113-151.

Berry, P.F. and Morgan, G.J. (1986). Decapod Crustacea of Scott and Seringapatam Reefs. In Berry, P.F. (ed.), Faunal surveys of the Rowley Shoals, Scott Reef and Seringapatam Reef, North-western Australia. Records of the Western Australian Museum 25: 59-62.
Black, R. and Prince, J. (1983). Fauna associated with the coral Pocillopora damicornis at the southern limit of its distribution in Western Australia. Journal of Biogeography 10: 135-152.

Castro, P. (1997a). Trapeziid crabs (Brachyura: Xanthoidea: Trapeziidae) of New Caledonia, eastern Australia, and the Coral Sea. In Richer de Forges, B. (ed.), Le benthos des fonds meubles des lagons de Nouvelle-Calédonie (sédimentologie, benthos). Études et Thèses 3: 59-107.

Castro, P. (1997b). Trapeziid crabs (Brachyura: Xanthoidea: Trapeziidae) of French Polynesia. In Richer de Forges, B. (ed.), Les fonds meubles des lagons de Nouvelle-Calédonie (Sédimentologie, Benthos). Etudes et Thèses 3: 109-139.

Castro, P. (1998). Systematic status and geographic distribution of Trapezia formosa Smith, 1869 (Crustacea, Brachyura, Trapeziidae), a symbiont of reef corals. Zoosystema 20: 177-181.

Castro, P. (1999a). Trapeziid crabs (Crustacea, Brachyura, Xanthoidea, Trapeziidae) of the Indian Ocean and the Red Sea. Zoosystema 21: 93-120.

Castro, P. (1999b). The Trapeziidae (Crustacea: Brachyura: Xanthoidea) of Indonesia, Results of the Rumphius Biohistorical Expedition to Ambon (1990), part 7. Zoologische Mededelingen 73: 27-61.

Castro, P. (2000). Biogeography of trapeziid crabs (Brachyura, Trapeziidae) symbiotic with reef corals and other cnidarians. In von Vaupel Klein, J.C. and Schram, F.R. (eds), The Biodiversity Crisis and Crustacea, Proceedings of the Fourth International Crustacean Congress, Amsterdam, vol. 2, Crustacean Issues 12: 65-75.

Castro, P. (2002). New records of trapeziid crabs (Xanthoidea, Trapeziidae) from the Andaman Sea coast of Thailand, with notes on the taxonomic status of Trapezia plana Ward, 1941. In Bruce, N.L. Berggren, M. and Bussawarit, S. (eds), Biodiversity of Crustacea of the Andaman Sea. Special Publication, Phuket Marine Biological Center 22: 361-367.

Castro, P. (2003). The trapeziid crabs (Brachyura) of Guam and Northern Mariana Islands, with the description of a new species of Trapezia Latreille, 1828. Micronesica 35-36: 440-455.

Castro, P., Ng, P.K.L. and Ahyong, S. (2004). Phylogeny and systematics of the Trapeziidae Miers, 1886 (Crustacea: Brachyura), with a description of a new family. Zootaxa 643:1-70.

Chen, P.S. (1933). Zur morphologie und Histologie der Respirations-organe von Grapsus grapsus L., nebst einer Liste Krabben der Sammlung Plate von Ceylon und Südindien. Jenaische Zeitschrift für Naturwissenschaften 68: 31-116.

Coulon, L. (1864). Crustacés de la Mer Rouge. Bulletin de la Société des Sciences Naturelles de Neuchâtel 6: 569-570.

Dana, J.D. (1851). On a new Genus of Crustacea in the Collections of the U.S. Exploring Expedition under Capt. C. Wilkes, U.S.N. American Journal of Science and Arts, ser. 2, 11: 223-224.

Dana, J.D. (1852a). Conspectus Crustaceorum, etc. Conspectus of the Crustacea of the Exploring 
Expedition under Capt. Wilkes, U.S.N., including the Crustacea Cancroidea Corystoidea. Proceedings of the Academy of Natural Sciences of Philadelphia 6: 73-86.

Dana, J.D. (1852b). Crustacea, Part I. United States Exploring Expedition, During the Years 1838, 1839, 1840, 1841, 1842, Under the Command of Charles Wilkes. U.S.N., Vol. 13. C. Sherman, Philadelphia, pp. 1-685. Atlas [1855]. 27 pp., pls. 1-96.

Davie, P.J.F. (2002). Crustacea: Malacostraca: Eucarida (Part 2): Decapoda-Anomura, Brachyura. In Wells, A. and Houston, W.W.K. (eds), Zoological Catalogue of Australia, 19.3B, CSIRO Publishing, Melbourne, xiv +641 pp.

Eydoux, F. and Souleyet, L. F. A. (1842). Crustacés. pp 219-272, atlas, pls 1-5. In, Voyage autour du monde exécuté pendant les années 1836 et 1837 sur la corvette la Bonite, commandée par $M$. Vaillant, Capitaine de Vaisseau, Zoologie. Paris. [For dates of publication see Sherborn \& Woodward, 1901: 391].

Forskål, P. (1775). Descriptiones Animalium Avium, Amphibiorum, Piscium, Insectorum, Vermium; quae in Itinere orientali observavit. Petrus Forskål. Post Mortem Auctoris editit Carsten Niebuhr. Adjuncta est materia Medica Kahirina. Hauniae, Heineck et Faber. pp. 1-9, i-xxxiv, 1-164, 1 map.

Galil, B. (1986a). Tetraloides - a new genus of coralinhabiting crabs. Crustaceana 50 [1985]: 68-77.

Galil, B. (1986b). Quadrella (Brachyura: Xanthoidea: Trapeziidae) - review and revision. Journal of Crustacean Biology 6: 275-293.

Galil, B. and Clark, P.F. (1988). On a collection of Acropora-inhabiting trapeziids (Crustacea Brachyura Xanthoidea) from East Africa. Tropical Zoology 1: 137-151.

Galil, B. and Clark, P.F. (1990). Crustacea Decapoda: Notes on trapeziid crabs from New Caledonia including descriptions of two new species. Pp. 369388, figs 1-6. In Crosnier, A. (ed.), Résultats des Campagnes MUSORSTOM. Volume 6. Mémoires $d u$ Muséum national d'Histoire naturelle, Paris (A) 145 : 1-388.

Garth, J.S. (1971). Borradaile's Maldivian collections revisited. Journal of the Marine Biological Association of India [1969] 11: 182-190.

Heller, C. (1861). Synopsis der im rothen Meere vorkommenden Crustaceen. Verhandlungen der Zoologisch-Botanischen Gesellschaft in Wien 11: 3-32.

Herbst, J.F.W. (1790). Versuch einer Naturgeschichte der Krabben und Krebse, nebst einer systematischen Beschreibung ihrer verschiedenen Arten. Berlin und Stralsund, Gottlieb, August und Lange. 1(8): 239-274, pls 18-21. [For dates of publication see Rathbun, 1897: 157]

Herbst, J.F.W. (1799). Versuch einer Naturgeschichte der Krabben und Krebse nebst einer Systematischen Beischreibung ihrer Verschieden Arten. Berlin and Stralsund : Gottlieb, August und Lange 3(1): 1-66 pls $47-50$

Herbst, J.F.W. (1801). Versuch einer Naturgeschichte der Krabben und Krebse, nebst einer systematischen Beschreibung ihrer verschiedenen Arten. Berlin und Stralsund, Gottlieb, August und Lange. 3(2): 1-46, pls
LI-LIV. [For dates of publication see Rathbun, 1897: 157]

Jacquinot, H. in Hombron, J.B. and Jacquinot, H. (1846). Atlas d'Histoire Naturelle Zoologie par MM. Hombron et Jacquinot, chirurgiens de l'expédition. In, Voyage au pôle sud et dans I'Océanie sur les Corvettes l'Astrolabe et la Zélée; exécuté par ordre du roi pendant les années 1837-1838-1839-1840 sous le commandement de $M$. Dumont d'Urville capitaine de vaisseau publié sous les auspices du département de la marine et sous la direction superieure de $M$. Jacquinot, capitaine de Vaisseau, commandant de La Zélée. Livraison 18. Oiseaux pl. 21; Crustacés pls 3, 4; Insectes coléoptères pls 16, 17. Paris, Gide et Cit.

Jones, D.S. (1990). Annotated checklist of marine decapod Crustacea from Shark Bay, Western Australia. In Berry, P.F., Bradshaw, S.D. and Wilson, B.R. (eds), Research in Shark Bay, Report of the France-Australe Bicentenary Expedition Committee: 169-208. Western Australian Museum, Perth.

Jones, D.S. and Morgan, G.J. (1993). An annotated checklist of Crustacea from Rottnest Island, Western Australia. In Wells, F.E., Walker, D.I., Kirkman, H. and Lethbridge, R. (eds), The Marine Flora and Fauna of Rottnest Island, Western Australia 1: 135-162. Western Australian Museum, Perth.

Jones, D.S. and Morgan, G.J. (1994). A Field Guide to Crustaceans of Australian Waters. Reed, Chatswood, Australia. 216 pp.

Jones, D.S. and Morgan, G.J. (2002). A Field Guide to Crustaceans of Australian Waters, $2^{\text {nd }}$ ed. Reed New Holland, Sydney. 224 pp.

Klunzinger, C.B. (1913). Die Rundkrabben (Cyclometpoa) des Roten Meeres. Nova Acta Academia Caesareae Leopoldino-Carolina Germanica Naturae Curiosorum 99: $103-402$.

Latreille, P.A. (1828). Art. Trichodactyle. In: Encyclopédie Méthodique: Entomologie, ou Histoire naturelle des Crutacés, des Arachinides et des Insectes. Paris, Chez Mme veune Agasse. [1825] 10: 705-706 [For dates of publication see Sherborn \& Woodward, 1899].

Laurie, R.D. (1915). On the Brachyura. Reports on the Marine Biology of the Sudanese Red Sea, from collections made by Cyril Crossland, M.A., B. Sc., F.Z.S., XXI. Journal of the Linnean Society of London, Zoology 31: 407-475.

Linnaeus, C. (1758). Systema Naturae per Regna Tria Naturae, Secundum Classes, Ordines, Genera, Species, cum Characteribus, Differentiis Synonymis, Locis Edition 10. Holmiae. 1: iii + 1-824

MacLeay, W.S. (1838). On the Brachyurous Decapod Crustacea brought from the Cape by Dr Smith. Pp. 53-71, pl. 3. In, Illustrations of the Annulosa of South Africa; being a portion of the objects of natural history collected during an expedition into the interior of South Africa, under the direction of Dr Andrew Smith, in the years, 1834, 1835, and 1836; fitted out by "The Cape of Good Hope Association for Exploring Central Africa". London. 75 pp, 4 pls.

Miers, E.J. (1886). Part II. - Report on the Brachyura collected by H.M.S. Challenger during the years 1873-76. In Report on the Scientific Results of the Voyage of H.M.S. Challenger during the years 1873 
1876 under the command of Captain George S. Nares, N.R., F.R.S. and the late Captain Frank Tourle Thomson, R.N., prepared under the Superintendence of the late Sir C. Wyville Thomson, Knt., F.R.S. \&c., Regius Professor of Natural history in the University of Edinburgh of the civilian scientific staff on board and now of John Murray one of the naturalists of the Expedition. Zoology, Published by Order of Her Majesty's Government. London, Edinburgh and Dublin, HMSO. 17: i-1 + 1-362, pls 1-29.

Milne Edwards, A. (1873). Recherches sur la faune carcinologique de la Nouvelle-Calédonie. Pt. 2. Groupe des Cyclometopes Neptunens. Nouvelle Archives du Muséum national d'Histoire naturelle, Paris 9: 155-332, pls 4-18.

Morgan, G.J. (1990). A collection of Thalassinidea, Anomura and Brachyura (Crustacea: Decapoda) from the Kimberley Region of northwestern Australia. Zoologische Verhandelingen 265: 1-90.

Odinetz, O.M. (1984). Révision des Trapezia du groupe cymodoce-ferruginea (Crustacea, Decapoda, Brachyura), avec des notes complémentaires concernant $T$. serenei Odinetz, 1983 et $T$. punctimanus Odinetz, 1983. Bulletin du Muséum national d'Histoire naturelle, Paris, (sect. A)6(2): 431-452.

Paul'son, O. (1875). Izsledovaniya rakoobraznykh krasnago morya szametkami otnositel'no rakoobraznykndrugikh morei, xiv + 1- 144p., pls 121 (1961, Studies on Crustacea of the Red Sea with notes regarding other seas. Part I. Podophthalmata and Edriophthalmata (Cumacea). English translation, Jerusalem, the Isreal Program for Scientific Translations, Translation by F. D. Pory, 1164, pls 1-21).

Rüppell, W.P.E.S. (1830). Beschreibung und Abbildung von 24 Arten kurzschwänzigen Krabben, als Beitrag zur Naturgeschichte des rothen Meeres. Frankfurt a.m., H.L. Brönner. 1-28, pls 1-6.

Serène, R. (1971). Observations on species of the group Trapezia rufopunctata-maculata, with a provisional key for all the species of Trapezia. Journal of the Marine Biological Association of India 11: 126-148.
Serène, R. (1984). Crustacés Décapodes Brachyoures de l'Océan Indien occidental et de la Mer Rouge, Xanthoidea: Xanthidae et Trapeziidae. Avec un addendum par Crosnier (A): Carpiliidae et Menippidae. Faune Tropicale XXIV: 1-349, figs A-C +1-243, pls 1-48.

Serène, R. and Dat, P.T. (1957). Note sur Tetralia nigrifrons Dana, 1952. Annales de la Faculté des Sciences, Saigon 27(107-121): figs 1-4, pls 1-3 [reprint 1-27].

Smith, S.I. (1869). Notes on new or little known species of American Cancroid Crustacea. Proceedings of the Boston Society of Natural History 12: 274-289.

Stimpson, W. (1858). Crustacea Cancroidea et Coryoidea: Prodromus descriptionis animalium evertebratorum, quae in Expeditione ad Oceanum Pacificum Septentrionalem, a Republica Federata missa, Cadwaladaro Ringgold et Johanne Rodgers Ducibus, observavit et descripsit W. Stimpson, Para IV. Proceedings of the Academy of Natural Sciences, Philadelphia 10: 31-40.

Stimpson, W. (1860). Notes on North American Crustacea, in the Museum of the Smithsonian Institution. No. II. Annals of the Lyceum of Natural History of New York 7: 177-246, pls 2, 5.

Veron, J.E.N. (1993). A biogeographic database of hermatypic coral species of the central Indo-Pacific, genera of the world. Australian Institute of Marine Science Monograph Series 10: 1-433.

Veron, J.E.N. and Marsh, L.M. (1988). Hermatypic corals of Western Australia: records and annotated list. Records of the Western Australian Museum Supplement 29: i-v, 1-136.

Ward, M. (1939). The Brachyura of the Second Templeton Crocker-American Museum Expedition to the Pacific Ocean. American Museum Novitates 1049: 1-15.

Ward, M. (1941). New Brachyura from the Gulf of Davao, Mindanao, Philippine Islands. American Museum Novitates, New York 1104: 1-15.

Ward, M. (1942). A new genus and eight new species of Brachyura from Mautitius and the Chagos Archipelago. Bulletin of the Mauritius Institute 2: $39-48$. 